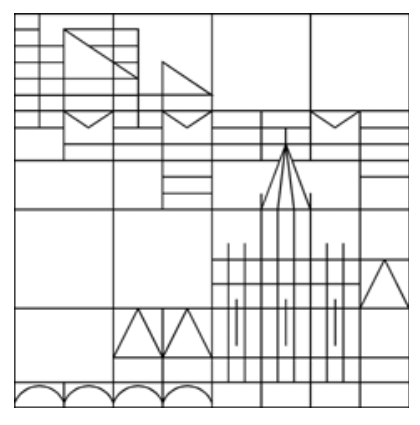

On exponential stability for thermoelastic plates : comparison and singular limits

\author{
Jaime E. Munoz Rivera \\ Reinhard Racke \\ Mauricio Sepulveda \\ Octavio Vera Villagran
}

Konstanzer Schriften in Mathematik

Nr. 374, März 2018

ISSN 1430-3558 



\title{
On exponential stability for thermoelastic plates: comparison and singular limits
}

\author{
J.E. Muñoz Rivera, R. Racke, M. Sepúlveda and O. Vera Villagrán
}

\begin{abstract}
We consider different models of thermoelastic plates in a bounded reference configuration: with Fourier heat conduction or with the Cattaneo model, and with or without inertial term. Some models exhibit exponential stability, others are not exponential stable. In the cases of exponential stability, we give an explicit estimate for the rate of decay in terms of the essential parameters appearing (delay $\tau \geq 0$, inertial constant $\mu \geq 0$ ). This is first done using multiplier methods directly in $L^{2}$-spaces, then, second, with eigenfunction expansions imitating Fourier transform techniques used for related Cauchy problems. The explicit estimates allow for a comparison. The singular limits $\tau \rightarrow 0$, and $\mu \rightarrow 0$ are also investigated in order to understand the mutual relevance for the (non-) exponential stability of the models. Numerical simulations underline the results obtained analytically, and exhibit interesting coincidences of analytical and numerical estimates, respectively.
\end{abstract}

\section{Introduction}

We consider the following system of thermoelastic plate equations,

$$
\begin{aligned}
\rho_{1} u_{t t}-\mu \Delta u_{t t}+\alpha \Delta^{2} u+\beta \Delta \theta & =0, \\
\rho_{2} \theta_{t}+\kappa \operatorname{div} q-\beta \Delta u_{t} & =0, \\
\tau q_{t}+\kappa_{0} q+\kappa_{1} \nabla \theta & =0 .
\end{aligned}
$$

Here $u, \theta:[0, \infty) \times \Omega \longrightarrow \mathbb{R}$ are the displacement and the temperature (difference to a fixed reference temperature), respectively, and $q:[0, \infty) \times \Omega \longrightarrow \mathbb{R}^{n}, n=1,2,3$, is the heat flux vector. $\Omega$ is assumed to be a smoothly bounded domain in $\mathbb{R}^{n}$. The constants $\rho_{1}, \alpha, \beta, \rho_{2}, \kappa, \kappa_{0}, \kappa_{1}$ are positive, while the constants $\tau$ and $\mu$, representing the delay and the inertial part, respectively, satisfy $\tau, \mu \geq 0$.

The system is completed by initial conditions,

$$
u(0, \cdot)=u^{0}, \quad u_{t}(0, \cdot)=u^{1}, \quad \theta(0, \cdot)=\theta^{0}, \quad \tau q(0, \cdot)=\tau q^{0},
$$

\footnotetext{
${ }^{0}$ AMS subject classification: 35 M 33, 35 B 40, 74 K 20, 74 F 05

Keywords and phrases: thermoelasticity, plates, Fourier law, Cattaneo law, inertial term, exponential stability, singular limit
} 
and by the following boundary conditions on $[0, \infty) \times \partial \Omega$,

$$
u=\Delta u=\theta=0
$$

We remark that the hinged boundary conditions for $u$ in (1.5) will allow in the sequel some calculations in Section 5 that do not follow this way for the Dirichlet boundary conditions

$$
u=\partial_{\nu} u=0,
$$

where $\partial_{\nu} u$ denotes the normal derivative on $\partial \Omega$. But the results on exponential stability and on the singular limit are expected to be qualitatively the same for both sets of boundary conditions. Hence our study provides the characteristic picture already in working with (1.5).

This system may represent different models for Kirchhoff type thermoelastic plate equations, with $(\mu>0)$ or without $(\mu=0)$ inertial term, either with Fourier's law $(\tau=0)$ of heat conduction or with Cattaneo's law $(\tau>0)$. We find the Schrödinger type part in (1.1), read mainly as equation for $u$, if $\mu=0$, a wave equation type if $\mu>0$, a parabolic equation in (1.2), (1.3) if $\tau=0$, resp. a hyperbolic system if $\tau>0$. This variety is reflected also in the different asymptotic behavior of the associated semigroups. We have

$$
\begin{aligned}
& \tau=0, \mu \geq 0: \text { exponential stability } \\
& \tau>0, \mu=0: \text { no exponential stability, } \\
& \tau>0, \mu>0: \text { exponential stability. }
\end{aligned}
$$

The aim is to prove the exponential stability results in a way that allows to give estimates on the expected stability constant $\gamma>0$ in the estimate

$$
\mathcal{E}(t) \leq K \mathrm{e}^{-\gamma t} \mathcal{E}(0)
$$

where $\mathcal{E}$ stands for the usual associated energy term. For example, the case $\tau>0, \mu>0$ was investigated in [3] with abstract semigroup theory, while we shall use an appropriate multiplier technique.

As a consequence, we shall obtain the dependence of the decay rate on $\tau$ and/or $\mu$, and we shall be able to compare the different models and to study the (singular) limits $\mu \rightarrow 0$ and $\tau \rightarrow 0$, respectively. The questions that we will address and answer are:

- How are the different parameters $\tau$ and $\mu$ reflected in the decay rates?

- Which models exhibit stronger/weaker decay? 
- How is the (singular) transition between the different models reflected in the estimates for the decay rates?

- How sharp are the analytical estimates obtained by energy (multiplier) methods (comparison with numerical results)?

The thermoelastic plate models provide interesting examples where, for $\mu=0$, exponential stability is lost when the Fourier law $(\tau=0)$ is replaced by the Cattaneo law $(\tau>0)$, see Quintanilla \& Racke [17] or Fernández Sare \& Muñoz Rivera [3]. A similar effect is know for certain Timoshenko systems, see Fernández Sare \& Racke [4], and these might not be isolated situations, see Racke [18]. On the other hand, as soon as the inertial term $\left(-\mu \Delta u_{t t}\right)$ is presented, exponential stability is given ([3]).

References for the exponential stability in the most explored case $\tau=\mu=0$ are, for example, Kim [6], Muñoz Rivera \& Racke [13, 14], Liu \& Zheng [12], Avalos \& Lasiecka [1], Lasiecka \& Triggiani $[7,8,9,10]$, for various boundary conditions and for the analyticity of the semigroups; see also [5] for a related system with memory term, and [2, 11, 16] for maximal regularity.

For the subsequent discussions we may assume w.l.o.g. for all constants different from $\tau$ and $\mu$, appearing in the differential equations,

$$
\rho_{1}=\alpha=\beta=\rho_{2}=\kappa=\kappa_{0}=\kappa_{1}=1 \text {. }
$$

The paper is organized as follows. In Section 2, we shall recall the exponential stability for the case $\tau=0, \mu=0$ by the multiplier method in $L^{2}$, with estimates on the decay rate. Section 3 presents the exponential stability for the case $\tau>0, \mu>0$, again with the multiplier method and with an estimate for the decay rate. In Section 4, we analyze the decay rates with respect to the singular limits $\mu \rightarrow 0$ and $\tau \rightarrow 0$. A second approach to obtain exponential stability and estimates for the decay rate is presented in Section 5 . Here we use an eigenfunction expansion (Fourier series) and exploit ideas from [19], where the corresponding Cauchy problem $\left(\Omega=\mathbb{R}^{n}\right)$ was investigated and where "energy" estimates were proved using the Fourier transform. In Section 6, an extended analysis of the singular limits is presented. Finally, in Section 7, we present a numerical analysis underlining the results obtained analytically in the previous sections. In particular, the numerical results perfectly correspond to the analytical estimates, demonstrating the sharpness of the latter. 


\section{Case $\tau=0, \mu=0$, multiplier method in $L^{2}$}

In this case with Fourier's law of heat conduction, and without inertial term, the differential equations (1.1)-(1.3) reduce to

$$
\begin{aligned}
u_{t t}+\Delta^{2} u+\Delta \theta & =0 \\
\theta_{t}-\Delta \theta-\Delta u_{t} & =0 .
\end{aligned}
$$

Defining the energy $E$ in this case of Fourier's law,

$$
E(t):=\frac{1}{2} \int_{\Omega}\left|u_{t}\right|^{2}+|\Delta u|^{2}+|\theta|^{2} d x
$$

and denoting $d_{t}:=\frac{d}{d t}$, we have

$$
d_{t} E(t)=-\int_{\Omega}|\nabla \theta|^{2} d x
$$

Standard multipliers are used. Multiplying (2.1) by $u$, assuming w.l.o.g. real-valued functions, and denoting by $\varepsilon_{j}>0, j \in \mathbb{N}$, small constants to be chosen later, we obtain

$$
\begin{aligned}
d_{t} \varepsilon_{1} \int_{\Omega} u_{t} u d x \leq & \varepsilon_{1} \int_{\Omega}\left|u_{t}\right|^{2} d x \\
& -\left(\varepsilon_{1}-c_{1} \varepsilon_{1} \varepsilon_{2}\right) \int_{\Omega}|\Delta u|^{2} d x \\
& +\frac{\varepsilon_{1}}{4 \varepsilon_{2}} \int_{\Omega}|\nabla \theta|^{2} d x,
\end{aligned}
$$

where $c_{j}>0, j \in \mathbb{N}$, will denote constants not depending $\varepsilon_{j}$. Multiplying (2.2) by $u_{t}$, we get

$$
\begin{aligned}
d_{t}\left(-\varepsilon_{3} \int_{\Omega} \theta u_{t} d x\right) \leq & -\varepsilon_{3} \int_{\Omega} \theta u_{t t} d x-\frac{\varepsilon_{3}}{2} \int_{\Omega}\left|\nabla u_{t}\right|^{2} d x+\frac{\varepsilon_{3}}{2} \int_{\Omega}|\nabla \theta|^{2} d x \\
\leq & -\varepsilon_{3} \int_{\Omega} \theta u_{t t} d x-\frac{\varepsilon_{3}}{4} \int_{\Omega}\left|\nabla u_{t}\right|^{2} d x-\frac{\varepsilon_{3} c_{p}}{4} \int_{\Omega}\left|u_{t}\right|^{2} d x \\
& +\frac{\varepsilon_{3}}{2} \int_{\Omega}|\nabla \theta|^{2} d x
\end{aligned}
$$

where here, and similarly in the sequel, $c_{p}$ denotes the constant appearing in the Poincaré estimate. Since

$$
\int_{\Omega} \theta u_{t t} d x=\int_{\Omega} \nabla \theta \nabla \Delta u d x+\left.\int_{\Omega} \nabla \theta\right|^{2} d x
$$

we have

$$
-\varepsilon_{3} \int_{\Omega} \theta u_{t t} d x \leq \frac{\varepsilon_{3}}{4 \varepsilon_{4}} \int_{\Omega}|\nabla \theta|^{2} d x+\varepsilon_{3} \varepsilon_{4} \int_{\Omega}|\nabla \Delta u|^{2} d x
$$


Combining (2.5), (2.6), we obtain

$$
\begin{aligned}
d_{t}\left(-\varepsilon_{3} \int_{\Omega} \theta u_{t} d x\right) \leq & \frac{\varepsilon_{3}}{4 \varepsilon_{4}} \int_{\Omega}|\nabla \theta|^{2} d x+\varepsilon_{3} \varepsilon_{4} \int_{\Omega}|\nabla \Delta u|^{2} d x-\frac{\varepsilon_{3}}{4} \int_{\Omega}\left|\nabla u_{t}\right|^{2} d x \\
& -\frac{\varepsilon_{3} c_{p}}{4} \int_{\Omega}\left|u_{t}\right|^{2} d x+\frac{\varepsilon_{3}}{2} \int_{\Omega}|\nabla \theta|^{2} d x .
\end{aligned}
$$

Finally multiplying (2.1) by $\Delta u$, we get

$$
d_{t}\left(-\varepsilon_{5} \int_{\Omega} u_{t} \Delta u d x\right) \leq \varepsilon_{5} \int_{\Omega}\left|\nabla u_{t}\right|^{2} d x-\frac{\varepsilon_{5}}{2} \int_{\Omega}|\nabla \Delta u|^{2} d x+\frac{\varepsilon_{5}}{2} \int_{\Omega}|\nabla \theta|^{2} d x .
$$

Defining the Lyapunov function $L$ by

$$
L(t):=M E(t)+\varepsilon_{1} \int_{\Omega} u_{t} u d x-\varepsilon_{3} \int_{\Omega} \theta u_{t} d x-\varepsilon_{5} \int_{\Omega} u_{t} \Delta u d x,
$$

with $M>0$ to be determined below, we obtain from (2.3), (2.4), (2.7), (2.8)

$$
\begin{aligned}
d_{t} L(t) \leq & -c_{p}\left(M-\frac{\varepsilon_{1}}{4 \varepsilon_{2}}-\frac{\varepsilon_{3}}{4 \varepsilon_{4}}-\frac{\varepsilon_{3}}{2}-\frac{\varepsilon_{5}}{2}\right) \int_{\Omega}|\theta|^{2} d x-\left(\frac{\varepsilon_{3} c_{p}}{4}-\varepsilon_{1}\right) \int_{\Omega}\left|u_{t}\right|^{2} d x \\
& -\left.\left(\frac{\varepsilon_{3}}{4}-\varepsilon_{5}\right) \int_{\Omega} \nabla u_{t}\right|^{2} d x-\left(\varepsilon_{1}-c_{1} \varepsilon_{1} \varepsilon_{2}\right) \int_{\Omega}|\Delta u|^{2} d x \\
& -\left(\frac{\varepsilon_{5}}{2}-\varepsilon_{3} \varepsilon_{4}\right) \int_{\Omega}|\nabla \Delta u|^{2} d x .
\end{aligned}
$$

Choosing $\varepsilon_{j}$ in the following way,

$$
\varepsilon_{3}:=1, \quad \varepsilon_{1}:=\frac{c_{p}}{8}, \quad \varepsilon_{5}:=\frac{1}{8}, \quad \varepsilon_{2}:=\frac{1}{2 c_{1}}, \quad \varepsilon_{4}:=\frac{1}{32},
$$

and

$$
M \geq 2 M_{1} \equiv 2\left(\frac{\varepsilon_{1}}{4 \varepsilon_{2}}+\frac{\varepsilon_{3}}{4 \varepsilon_{4}}+\frac{\varepsilon_{3}}{2}+\frac{\varepsilon_{5}}{2}\right)=\frac{c_{1}+548}{32}
$$

we have

$$
\begin{aligned}
d_{t} L(t) & \leq-c_{p} M_{1} \int_{\Omega}|\nabla \theta|^{2} d x-\frac{c_{p}}{4} \int_{\Omega}\left|u_{t}\right|^{2} d x-\frac{c_{p}}{8} \int_{\Omega}|\Delta u|^{2} d x \\
& \leq-d_{0} E(t),
\end{aligned}
$$

with

$$
d_{0}:=2 c_{p} \min \left\{M_{1}, \frac{1}{8}\right\}
$$

To assure the equivalence of $E(t)$ and $L(t)$, we compute

$$
\begin{aligned}
&\left|\varepsilon_{1} \int_{\Omega} u_{t} u d x-\varepsilon_{3} \int_{\Omega} \theta u_{t} d x-\varepsilon_{5} \int_{\Omega} u_{t} \Delta u d x\right| \\
&\left(\frac{c_{p}+9}{8}\right) \frac{1}{2} \int_{\Omega}\left|u_{t}\right|^{2} d x+\left(\frac{c_{p} c_{2}+1}{8}\right) \frac{1}{2} \int_{\Omega}|\Delta u|^{2} d x+\frac{1}{2} \int_{\Omega}|\theta|^{2} d x
\end{aligned}
$$




$$
\leq \max \left\{\frac{c_{p}+9}{8}, \frac{c_{p} c_{2}+1}{8}, 1\right\} E(t) \equiv M_{2} E(t),
$$

where $c_{2}$ arises from the elliptic estimate

$$
\int_{\Omega}|u|^{2} d x \leq c_{2} \int_{\Omega}|\Delta u|^{2} d x .
$$

This implies, choosing

$$
M \geq 2 M_{2},
$$

the equivalence, for $t \geq 0$,

$$
k_{1} E(t) \leq L(t) \leq k_{2} E(t)
$$

with

$$
k_{1}:=M_{2}, \quad k_{2}:=3 M_{2} .
$$

Altogether, we have with

$$
M:=\max \left\{2 M_{1}, M_{2}\right\}
$$

and by (2.12), (2.15),

$$
d_{t} L(t) \leq-\frac{d_{0}}{k_{2}} L(t)
$$

Defining

$$
K_{0}:=\frac{k_{2}}{k_{1}}, \quad \gamma_{0}:=\frac{d_{0}}{k_{2}},
$$

we have the following exponential stability result

Theorem 2.1. The inital-boundary value problem (2.1), (2.2), (1.4), (1.5) is exponentially stable. We have for $t \geq 0$ the following estimate for the associated energy,

$$
E(t) \leq K_{0} \mathrm{e}^{-\gamma_{0} t} E(0) .
$$

The constant $\gamma_{0}$ is given explicitly through (2.17), (2.16), (2.13), (2.11), and the type $\omega_{0}$ of the semigroup is hence estimated from above by

$$
\omega_{0} \leq-\gamma_{0}
$$

\section{Case $\tau>0, \mu>0$, multiplier method in $L^{2}$}

In the case of Cattaneo's law of heat conduction and with intertial term, we shall prove a similar result on exponential stability as in Theorem 2.1, but now with estimates on the rate of decay $\gamma=\gamma(\mu, \tau)$. We consider

$$
\begin{aligned}
u_{t t}-\mu \Delta u_{t t}+\Delta^{2} u+\Delta \theta & =0 \\
\theta_{t}+\operatorname{div} q-\Delta u_{t} & =0 \\
\tau q_{t}+q+\nabla \theta & =0
\end{aligned}
$$


together with the initial conditions (1.4) and the boundary conditions (1.5). Let

$$
\mathcal{E}_{1}(t):=\frac{1}{2} \int_{\Omega}\left|u_{t}\right|^{2}+\mu\left|\nabla u_{t}\right|^{2}+|\Delta u|^{2}+|\theta|^{2}+\tau|q|^{2} d x
$$

and

$$
\mathcal{E}_{2}(t):=\frac{1}{2} \int_{\Omega}\left|u_{t t}\right|^{2}+\mu\left|\nabla u_{t t}\right|^{2}+\left|\Delta u_{t}\right|^{2}+\left|\theta_{t}\right|^{2}+\tau\left|q_{t}\right|^{2} d x
$$

denote the energies of first and of second order, respectively, and let

$$
\mathcal{E}(t):=\mathcal{E}_{1}(t)+\mathcal{E}_{2}(t)
$$

denote the energy, for which we shall prove a result on the exponential decay, in which we shall be able to observe the dependence of the estimate for the decay rate on the parameters $\mu$, in particular, as well as on $\tau$. We assume w.l.o.g. $\tau<1$ ( $\tau \geq 1$ could be treated similarly). We have

$$
d_{t} \mathcal{E}_{1}(t)=-\int_{\Omega}|q|^{2} d x, \quad d_{t} \mathcal{E}_{2}(t)=-\int_{\Omega}\left|q_{t}\right|^{2} d x
$$

implying

$$
d_{t} \mathcal{E}(t) \leq-\frac{1}{2 \tau} \int_{\Omega} \tau|q|^{2} d x-\left(\frac{2-\tau^{2}}{2 \tau}\right) \int_{\Omega} \tau\left|q_{t}\right|^{2} d x-\frac{1}{4} \int_{\Omega}|\nabla \theta|^{2} d x .
$$

Multiplying (3.1) by $\Delta u$, we obtain

$$
\begin{aligned}
d_{t}\left(-\delta_{1} \int_{\Omega}\left(u_{t}+\mu \Delta u_{t}\right) \Delta u d x\right) \leq & -\frac{\delta_{1}}{2} \int_{\Omega}|\nabla \Delta u|^{2} d x-\delta_{1} \int_{\Omega}\left|\Delta u_{t}\right|^{2} d x \\
& +\frac{\delta_{1}}{2} \int_{\Omega}|\nabla \theta|^{2} d x+\frac{\delta_{1}}{\mu} \mu \int_{\Omega}\left|\nabla u_{t}\right|^{2} d x
\end{aligned}
$$

where $\delta_{j}, j \in \mathbb{N}$, will denote positive constants to be chosen later. Multiplying (3.2) by $u_{t}$, we get

$$
\begin{aligned}
d_{t} \int_{\Omega} \theta u_{t} d x \leq & \frac{1}{4 \delta_{2}} \int_{\Omega}|\nabla \theta|^{2} d x+\delta_{2} \int_{\Omega}|\nabla \Delta u|^{2} d x+\frac{\mu}{4 \delta_{3}} \int_{\Omega}|\nabla \theta|^{2} d x \\
& +\delta_{3} \mu \int_{\Omega}\left|\nabla u_{t t}\right|^{2} d x+\int_{\Omega}|\nabla \theta|^{2} d x+\frac{1}{2} \int_{\Omega}|q|^{2} d x-\frac{1}{2} \int_{\Omega}\left|\nabla u_{t}\right|^{2} d x,
\end{aligned}
$$

implying

$$
\begin{aligned}
d_{t}\left(\delta_{4} \int_{\Omega} \theta u_{t} d x\right) \leq & \left(\frac{\delta_{4}}{4 \delta_{2}}+\frac{\delta_{4} \mu}{4 \delta_{3}}+\delta_{4}\right) \int_{\Omega}|\nabla \theta|^{2} d x+\delta_{2} \delta_{4} \int_{\Omega}|\nabla \Delta u|^{2} d x \\
& +\delta_{3} \delta_{4} \mu \int_{\Omega}\left|\nabla u_{t t}\right|^{2} d x+\frac{\delta_{4}}{2 \tau} \int_{\Omega} \tau|q|^{2} d x \\
& -\frac{\delta_{4}}{2 \mu} \mu \int_{\Omega}\left|\nabla u_{t}\right|^{2} d x .
\end{aligned}
$$


In order to to obtain negative terms for $u_{t t}$ and $\nabla u_{t t}$, we multiply (3.1) by $u_{t t}$ and obtain

$$
\begin{aligned}
d_{t}\left(\delta_{5} \int_{\Omega} \Delta u \Delta u_{t} d x\right) \leq & -\delta_{5} \int_{\Omega}\left|u_{t t}\right|^{2} d x-\frac{\delta_{5} \mu}{2} \int_{\Omega}\left|\nabla u_{t t}\right|^{2} d x+\delta_{5} \int_{\Omega}\left|\Delta u_{t}\right|^{2} d x \\
& +\frac{\delta_{5}}{2 \mu} \int_{\Omega}|\nabla \theta|^{2} d x .
\end{aligned}
$$

Finally we multiply (3.2) by $\theta_{t}$ and get

$$
\begin{aligned}
d_{t}\left(\delta_{6} \int_{\Omega} q \nabla \theta d x\right) \leq & -\frac{\delta_{6}}{2} \int_{\Omega}\left|\theta_{t}\right|^{2} d x+\frac{\delta_{6}}{2} \int_{\Omega}\left|\Delta u_{t}\right|^{2} d x+\frac{1}{2 \tau} \int_{\Omega} \tau|q|^{2} d x \\
& +\frac{\delta_{6}}{2} \int_{\Omega}|\nabla \theta|^{2} d x .
\end{aligned}
$$

We define the Lyapunov functional $\mathcal{L}$ by

$$
\begin{aligned}
\mathcal{L}(t):= & P \mathcal{E}(t)-\delta_{1} \int_{\Omega}\left(u_{t}+\mu \Delta u_{t}\right) \Delta u d x+\delta_{4} \int_{\Omega} \theta u_{t} d x \\
& +\delta_{5} \int_{\Omega} \Delta u \Delta u_{t} d x+\delta_{6} \int_{\Omega} q \nabla \theta d x,
\end{aligned}
$$

where also $P>0$ will have to be chosen appropriately later on. The estimates $(3.4)-(3.8)$ imply

$$
\begin{aligned}
d_{t} \mathcal{L}(t) \leq & -\left(\frac{P}{4}-\frac{\delta_{1}}{2}-\frac{\delta_{4} \mu}{4 \delta_{2}}-\frac{\delta_{4} \mu}{4 \delta_{3}}-\delta_{4}-\frac{\delta_{5}}{2 \mu}-\frac{\delta_{6}}{2}\right) \int_{\Omega}|\nabla \theta|^{2} d x \\
& -P\left(\frac{2-\tau^{2}}{2 \tau}\right) \int_{\Omega} \tau\left|q_{t}\right|^{2} d x-\left(\frac{P}{2 \tau}-\frac{\delta_{4}}{2 \tau}-\frac{1}{2 \tau}\right) \int_{\Omega} \tau|q|^{2} d x \\
& -\left(\frac{\delta_{1}}{2}-\delta_{2} \delta_{4}\right) \int_{\Omega}|\nabla \Delta u|^{2} d x-\left(\delta_{1}-\delta_{5}-\frac{\delta_{6}}{2}\right) \int_{\Omega}\left|\Delta u_{t}\right|^{2} d x \\
& -\left(\frac{\delta_{4}}{2 \mu}-\frac{\delta_{1}}{\mu}\right) \int_{\Omega} \mu\left|\nabla u_{t}\right|^{2} d x-\delta_{5} \int_{\Omega}\left|u_{t t}\right|^{2} d x \\
& -\left(\frac{\delta_{5}}{2}-\delta_{3} \delta_{4}\right) \int_{\Omega} \mu\left|\nabla u_{t t}\right|^{2} d x-\frac{\delta_{6}}{2} \int_{\Omega}\left|\theta_{t}\right|^{2} d x .
\end{aligned}
$$

Choosing $\delta_{j}$ in the following way,

$$
\delta_{4}:=\mu, \quad \delta_{1}:=\frac{\mu}{4}, \quad \delta_{5}:=\frac{\mu}{16}, \quad \delta_{6}:=\frac{\mu}{8}, \quad \delta_{2}:=\frac{1}{16}, \quad \delta_{3}:=\frac{1}{64},
$$

and

$$
P \geq P_{1}:=\frac{1}{4}\left(1+166 \mu+512 \mu^{2}\right),
$$


we conclude

$$
\begin{aligned}
d_{t} \mathcal{L}(t) \leq & -\frac{1}{32}\left(1+166 \mu+512 \mu^{2}\right) \int_{\Omega}|\nabla \theta|^{2} d x \\
& -P\left(\frac{2-\tau^{2}}{2 \tau}\right) \int_{\Omega} \tau\left|q_{t}\right|^{2} d x-\frac{1+\mu}{2 \tau} \int_{\Omega} \tau|q|^{2} d x \\
& -\frac{\mu}{16} \int_{\Omega}|\nabla \Delta u|^{2} d x-\frac{\mu}{8} \int_{\Omega}\left|\Delta u_{t}\right|^{2} d x-\frac{1}{4} \int_{\Omega} \mu\left|\nabla u_{t}\right|^{2} d x \\
& -\frac{\mu}{16} \int_{\Omega}\left|u_{t t}\right|^{2} d x-\frac{\mu}{64} \int_{\Omega} \mu\left|\nabla u_{t t}\right|^{2} d x-\frac{\mu}{16} \int_{\Omega}\left|\theta_{t}\right|^{2} d x \\
\leq & -\frac{c_{p}}{32}\left(1+166 \mu+512 \mu^{2}\right) \int_{\Omega}|\theta|^{2} d x \\
& -P\left(\frac{2-\tau^{2}}{2 \tau}\right) \int_{\Omega} \tau\left|q_{t}\right|^{2} d x-\frac{1+\mu}{2 \tau} \int_{\Omega} \tau|q|^{2} d x-\frac{c_{p} \mu}{16} \int_{\Omega}|\Delta u|^{2} d x \\
& -\frac{\mu}{8} \int_{\Omega}\left|\Delta u_{t}\right|^{2} d x-\frac{1}{8} \int_{\Omega} \mu\left|\nabla u_{t}\right|^{2} d x-\frac{c_{p} \mu}{8} \int_{\Omega}\left|u_{t}\right|^{2} d x \\
& -\frac{\mu}{16} \int_{\Omega}\left|u_{t t}\right|^{2} d x-\frac{\mu}{64} \int_{\Omega} \mu\left|\nabla u_{t t}\right|^{2} d x-\frac{\mu}{16} \int_{\Omega}\left|\theta_{t}\right|^{2} d x \\
\leq & -d \mathcal{E}(t),
\end{aligned}
$$

with

$$
d:=2 \min \left\{\frac{c_{p}}{32}\left(1+166 \mu+512 \mu^{2}\right), P\left(\frac{2-\tau^{2}}{\tau}\right), \frac{1+\mu}{\tau}, \frac{c_{p} \mu}{4}, \frac{1}{4}, \frac{\mu}{32},\right\} .
$$

The equivalence of $\mathcal{L}(t)$ and $\mathcal{E}(t)$ is given as follows, using

$$
\begin{gathered}
\int_{\Omega} q \nabla \theta d x=\int_{\Omega}\left(\theta_{t}-\Delta u_{t}\right) \theta d x \\
\left|-\delta_{1} \int_{\Omega}\left(u_{t}+\mu \Delta u_{t}\right) \Delta u d x+\delta_{4} \int_{\Omega} \theta u_{t} d x+\delta_{5} \int_{\Omega} \Delta u \Delta u_{t} d x+\delta_{6} \int_{\Omega} q \nabla \theta d x\right| \\
\leq \frac{3 \mu}{4} \int_{\Omega}\left|u_{t}\right|^{2} d x+\left(\frac{\mu^{3}}{8}+\frac{5 \mu}{32}\right) \int_{\Omega}\left|\Delta u_{t}\right|^{2} d x+\frac{5 \mu}{32} \int_{\Omega}|\Delta u|^{2} d x \\
+\frac{9}{16} \int_{\Omega}|\theta|^{2} d x+\frac{\mu}{8} \int_{\Omega}\left|\theta_{t}\right|^{2} d x \\
\leq \max \left\{\frac{3 \mu}{2}, \frac{\mu^{3}}{4}+\frac{5 \mu}{16}, \frac{9}{8}\right\} \mathcal{E}(t) \equiv P_{2} \mathcal{E}(t) .
\end{gathered}
$$

Choosing

$$
P \geq 2 P_{2}
$$

the equivalence, for $t \geq 0$,

$$
p_{1} \mathcal{E}(t) \leq \mathcal{L}(t) \leq p_{2} \mathcal{E}(t)
$$


is given with

$$
p_{1}:=P_{2}, \quad p_{2}:=3 P_{2}
$$

Choosing

$$
P:=\max \left\{2 P_{1}, P_{2}\right\}
$$

and by (3.11), (3.15), we obtain

$$
d_{t} L(t) \leq-\frac{d}{p_{2}} L(t)
$$

Defining

$$
K:=\frac{p_{2}}{p_{1}}, \quad \gamma:=\frac{d}{p_{2}},
$$

we have the following exponential stability result:

Theorem 3.1. The inital-boundary value problem (3.1)-(3.3), (1.4), (1.5) is exponentially stable. We have for $t \geq 0$ the following estimate for the associated energy,

$$
\mathcal{E}(t) \leq K \mathrm{e}^{-\gamma t} \mathcal{E}(0)
$$

The constant $\gamma=\gamma(\mu, \tau)$ is given explicitly, see (3.17), (3.16), (3.14), (3.13), (3.12), (3.10), and the type $\omega$ of the semigroup is hence estimated from above by

$$
\omega \leq-\gamma
$$

\section{The limits $\mu \rightarrow 0$ and $\tau \rightarrow 0$}

The rate of exponential stability, $\gamma=\gamma(\mu, \tau)$, given in Theorem 3.1, can now be studied with respect to the limits $\mu \rightarrow 0$ and $\tau \rightarrow 0$, respectively. We have

Theorem 4.1. The rate of exponential stability $\gamma$ given in Theorem 3.1 satisfies:

1. For fixed $\tau>0$ we have

$$
\lim _{\mu \rightarrow 0} \gamma(\mu, \tau)=0
$$

2. For fixed $\mu>0$ we have

$$
\lim _{\tau \rightarrow 0} \gamma(\mu, \tau)=c(\mu)
$$

where $c(\mu)$ is a positive constant depending on $\mu$.

Proof: The representation for $\gamma$ is given by (3.10), (3.12), (3.13), (3.16), (3.17),

$$
\gamma=\frac{d}{p_{2}}=\frac{2 \min \left\{\frac{c_{p}}{32}\left(1+166 \mu+512 \mu^{2}\right), P\left(\frac{2-\tau^{2}}{\tau}\right), \frac{1+\mu}{\tau}, \frac{c_{p} \mu}{4}, \frac{1}{4}, \frac{\mu}{32},\right\}}{3 \max \left\{\frac{3 \mu}{2}, \frac{\mu^{3}}{4}+\frac{5 \mu}{16}, \frac{9}{8}\right\}} .
$$


Observing that for sufficiently small $\mu$

$$
P=\frac{9}{8}
$$

we immediately conlude assertion 1 . The second assertion follows observing that $P$ is only depending on $\mu$. This completes the proof.

Theorem 4.1 reflects the facts that for $\mu \rightarrow 0$, the exponential stability is lost, and that the limit $\tau \rightarrow 0$ leads to another exponentially stable system.

The advantage of the multiplier method in $L^{2}$ is that it can be extended to other boundary conditions like (1.6), a disadvantage consists in the still coarse estimates leading to overall too pessimistic values e.g. for $c(\mu)$. Since $c(\mu) \rightarrow 0$ as $\mu \rightarrow 0$ it does - in the double limit - not reflect the fact that the system for $\mu=\tau=0$ is exponentially stable.

A sharper analysis, but then only for the hinged boundary conditions, will be given starting in the next section.

\section{Case $\tau>0, \mu>0$, Fourier expansion}

As second approach to obtain exponential stability and estimates on the decay rates, we make the following Fourier series ansatz. It will give us explicit information on the dependence of the decay rates on the parameters $\mu$ and $\tau$, but also on the domain $\Omega$ (appearing in form of the first eigenvalue $\lambda_{1}$ of the Dirichlet Laplace operator and depending itself on the size of the domain).

To justify the special ansatz below for the heat flux $q$, we assume the compatibility condition

$$
q^{0}=-\nabla \theta^{0}
$$

which is satisfied for $\tau=0$ anyway and, hence, avoids layers in the singular limit $\tau \rightarrow 0$. Then $q=q(t, \cdot)$ is a gradient field for all $t \geq 0$ by (1.3).

Let $\left(\phi_{j}\right)_{j}$ denote the $L^{2}$-eigenfunctions of the Dirichlet Laplace operator $-\Delta$ in $\Omega$ with eigenvalues $\left(\lambda_{j}\right)_{j}$ satisfying

$$
-\Delta \phi_{j}=\lambda_{j} \phi_{j}, \quad \phi_{j \mid \partial \Omega}=0, \quad 0<\lambda_{1} \leq \lambda_{2} \leq \cdots \leq \lambda_{j} \rightarrow \infty \quad(j \rightarrow \infty) .
$$

We make the following ansatz which is justified because of the boundary conditions (1.5).

$$
u(t, x)=\sum_{j=1}^{\infty} a_{j}(t) \phi_{j}(x), \quad \theta(t, x)=\sum_{j=1}^{\infty} b_{j}(t) \phi_{j}(x), \quad q(t, x)=\sum_{j=1}^{\infty} f_{j}(t) \nabla \phi_{j}(x) .
$$


Plugging this ansatz into the differential equations (1.1)-(1.3), we obtain the following system of ODEs for the coefficient functions $a_{j}, b_{j}, f_{j}$ :

$$
\begin{aligned}
\left(1+\mu \lambda_{j}\right) a_{j}^{\prime \prime}(t)+\lambda_{j}^{2} a_{j}(t)-\lambda_{j} b_{j}(t) & =0, \\
b_{j}^{\prime}(t)-\lambda_{j} f_{j}(t)+\lambda_{j} a_{j}^{\prime}(t) & =0, \\
\tau f_{j}^{\prime}(t)+f_{j}(t)+b_{j}(t) & =0 .
\end{aligned}
$$

In order to be able to compare it to the results obtained in the recent paper [19] on the Cauchy problem, we define

$$
d_{j}(t):=i \sqrt{\lambda_{j}} f_{j}(t)
$$

giving

$$
-\lambda_{j} f_{j}(t)=i \sqrt{\lambda_{j}} d_{j}(t)
$$

Multiplying the differential equation (5.6) by $i \sqrt{\lambda_{j}}$ we thus obtain the following system of ODEs for $a_{j}, b_{j}, d_{j}$ :

$$
\begin{aligned}
\left(1+\mu \lambda_{j}\right) a_{j}^{\prime \prime}(t)+\lambda_{j}^{2} a_{j}(t)-\lambda_{j} b_{j}(t) & =0 \\
b_{j}^{\prime}(t)+i \sqrt{\lambda_{j}} d_{j}(t)+\lambda_{j} a_{j}^{\prime}(t) & =0 \\
\tau d_{j}^{\prime}(t)+d_{j}(t)+i \sqrt{\lambda_{j}} b_{j}(t) & =0 .
\end{aligned}
$$

In [19] the Cauchy problem $\left(\Omega=\mathbb{R}^{n}\right)$ for the equations (1.1)-(1.3) was analyzed. Using the Fourier transform $(x \rightarrow \xi)$, the Fourier transformed functions $\hat{u}, \hat{\theta}, \hat{q}$, depending on $t, \xi$, satisfy

$$
\begin{aligned}
\left(1+\mu|\xi|^{2}\right) \hat{u}_{t t}+|\xi|^{4} \hat{u}-|\xi|^{2} \hat{\theta} & =0, \\
\hat{\theta}_{t}+i \xi \cdot \hat{q}+|\xi|^{2} \hat{u}_{t} & =0, \\
\tau \hat{q}_{t}+\hat{q}+i \xi \hat{\theta} & =0 .
\end{aligned}
$$

Identifying - formally $-\lambda_{j}$ with $|\xi|^{2}$, as well as $\sqrt{\lambda_{j}}$ with $\xi$, the similarities between the equations (5.6)-(5.8) and (5.9)-(5.11) are obvious. Actually, in one space dimension, $\sqrt{\lambda_{j}}$ and $\xi$ correspond perfectly for what follows.

It turns out that the series of estimates obtained in [19] for the "energy" term

$$
\left(1+\mu|\xi|^{2}\right)\left|\hat{u}_{t}(t, \xi)\right|^{2}+|\xi|^{4}|\hat{u}(t, \xi)|^{2}+|\hat{\theta}(t, \xi)|^{2}+\tau|\hat{q}(t, \xi)|^{2}
$$

can be carried over to the "energy" term

$$
\left(1+\mu \lambda_{j}\right)\left|a_{j}^{\prime}(t)\right|^{2}+\lambda_{j}^{2}\left|a_{j}(t)\right|^{2}+\left|b_{j}(t)\right|^{2}+\tau\left|d_{j}(t)\right|^{2} .
$$

Since we are later on interested in the limit $\mu \rightarrow 0$ and $\tau \rightarrow 0$, we assume from now on

$$
\tau, \mu \leq 1
$$

So we obtain from the proof of [19, Theorem 4.1] 
Theorem 5.1. There are constants $C, c_{1}>0$ (in particular neither depending on the parameters $\mu, \tau$ and $j$ nor on the data) such that for all $t \geq 0$ and all $j \in \mathbb{N}$ the estimate

$$
\begin{aligned}
& \left(1+\mu \lambda_{j}\right)\left|a_{j}^{\prime}(t)\right|^{2}+\lambda_{j}^{2}\left|a_{j}(t)\right|^{2}+\left|b_{j}(t)\right|^{2}+\tau\left|d_{j}(t)\right|^{2} \leq \\
& C \mathrm{e}^{-c_{1} \varrho_{j} t}\left(\left(1+\mu \lambda_{j}\right)\left|a_{j}^{\prime}(0)\right|^{2}+\lambda_{j}^{2}\left|a_{j}(0)\right|^{2}+\left|b_{j}(0)\right|^{2}+\tau\left|d_{j}(0)\right|^{2}\right)
\end{aligned}
$$

holds, where

$$
\varrho_{j} \equiv \varrho_{j}(\mu, \tau):=\frac{\lambda_{j}\left(1+\tau \mu \lambda_{j}\right)}{\left(1+\tau \lambda_{j}\right)\left(1+(\tau+\mu) \lambda_{j}\right)} .
$$

The constants $C, c_{1}$ are given explicitly by

$$
C=\frac{13}{11}, \quad c_{1}=\frac{1}{2730} .
$$

For the convenience of the reader, we present a sketch of the PROOF, for more details cp. [19]. We obtain from the differenatial equations (5.6)-(5.8) for

$$
W_{j}(t):=\left(1+\mu \lambda_{j}\right)\left|a_{j}^{\prime}(t)\right|^{2}+\lambda_{j}^{2}\left|a_{j}(t)\right|^{2}+\left|b_{j}(t)\right|^{2}+\tau\left|d_{j}(t)\right|^{2}
$$

that

$$
\frac{1}{2} \frac{d}{d t} W_{j}(t)+\left|d_{j}(t)\right|^{2}=0 .
$$

Choosing appropriate multipliers for the equations (5.6)-(5.8), we get

$$
\frac{d}{d t} E_{j}^{1}(t)+D_{j}^{1}(t)=0
$$

where

$$
\begin{aligned}
E_{j}^{1}(t): & =\frac{1}{2}\left(1+\tau \lambda_{j}\right)\left(1+(\tau+\mu) \lambda_{j}\right)\left\{\left(1+\mu \lambda_{j}\right)\left|a_{j}^{\prime}(t)\right|^{2}+\lambda_{j}^{2}\left|a_{j}(t)\right|^{2}+\left|b_{j}(t)\right|^{2}+\tau\left|d_{j}(t)\right|^{2}\right\} \\
& +\alpha_{1}\left(1+\tau \mu \lambda_{j}\right)\left\{\tau \sqrt{\lambda_{j}} \cdot \operatorname{Re}\left(i b_{j}(t) \overline{d_{j}(t)}\right)+\alpha_{2}\left(1+\mu \lambda_{j}\right)\left(\operatorname{Re}\left(a_{j}^{\prime}(t) \overline{b_{j}(t)}\right)\right.\right. \\
& \left.\left.+\alpha_{3} \lambda_{j} \operatorname{Re}\left(a_{j}^{\prime}(t) \overline{a_{j}(t)}\right)\right)\right\} \\
D_{j}^{1}(t): & =\alpha_{1} \alpha_{2}\left(1-\alpha_{3}\right)\left(1+\tau \mu \lambda_{j}\right)\left(1+\mu \lambda_{j}\right) \lambda_{j}\left|a_{j}(t)\right|^{2}+\alpha_{1} \alpha_{2} \alpha_{3}\left(1+\tau \mu \lambda_{j}\right) \lambda_{j}^{3}\left|a_{j}(t)\right|^{2} \\
& +\alpha_{1}\left(1-\alpha_{2}\right)\left(1+\tau \mu \lambda_{j}\right) \lambda_{j}\left|b_{j}(t)\right|^{2}+\left|d_{j}(t)\right|^{2}+(2 \tau+\mu) \lambda_{j}\left|d_{j}(t)\right|^{2}-\alpha_{1} \tau\left|\sqrt{\lambda_{j}} d_{j}(t)\right|^{2} \\
& +\tau(\tau+\mu) \lambda_{j}^{2}\left|d_{j}(t)\right|^{2}-\alpha_{1} \tau^{2} \mu \lambda_{j}\left|\sqrt{\lambda_{j}} d_{j}(t)\right|^{2} \\
& +\alpha_{1} \alpha_{2}\left(1-\alpha_{3}\right)\left(1+\tau \mu \lambda_{j}\right) \lambda_{j}^{2} \operatorname{Re}\left(a_{j}(t) \overline{b_{j}(t)}\right) \\
& +\alpha_{1}\left(1+\tau \mu \lambda_{j}\right) \sqrt{\lambda_{j}} \operatorname{Re}\left(i b_{j}(t) \overline{d_{j}(t)}\right) \\
& -\alpha_{1}\left\{\alpha_{2}-\left(\tau-\alpha_{2} \mu\right) \lambda_{j}\right\}\left(1+\tau \mu \lambda_{j}\right) \sqrt{\lambda_{j}} \operatorname{Re}\left(i a_{j}(t)_{t} \overline{d_{j}(t)}\right) .
\end{aligned}
$$

The positive constants $\alpha_{1}, \alpha_{2}, \alpha_{3}$ are chosen small enough in the course of the proof. Then one concludes

$$
\begin{aligned}
D_{j}^{1}(t) \geq \frac{1}{7 !} & \left(1+\tau \mu \lambda_{j}\right) \lambda_{j}\left\{\left(1+\mu \lambda_{j}\right)\left|a_{j}^{\prime}(t)\right|^{2}+\lambda_{j}^{2}\left|a_{j}(t)\right|^{2}+\left|b_{j}(t)\right|^{2}\right\} \\
& +\frac{1}{16}\left(1+\tau \lambda_{j}\right)\left(1+(\tau+\mu) \lambda_{j}\right)\left|d_{j}(t)\right|^{2},
\end{aligned}
$$


as well as

$$
\begin{aligned}
& E_{j}^{1}(t) \leq \frac{13}{24}\left(1+\tau \lambda_{j}\right)\left(1+(\tau+\mu) \lambda_{j}\right) W_{j}(t), \\
& E_{j}^{1}(t) \geq \frac{11}{24}\left(1+\tau \lambda_{j}\right)\left(1+(\tau+\mu) \lambda_{j}\right) W_{j}(t) .
\end{aligned}
$$

Applying the estimates (5.17) and (5.18) to (5.16) we get

$$
\frac{d}{d t} E_{j}^{1}(t)+\frac{1}{2730} \frac{\left(1+\tau \mu \lambda_{j}\right)|\xi|^{2}}{\left(1+\tau \lambda_{j}\right)\left(1+(\tau+\mu) \lambda_{j}\right)} E_{j}^{1}(t) \leq 0 .
$$

This gives

$$
W_{j}(t) \leq \frac{13}{11} e^{-\frac{1}{2730} \frac{\left(1+\tau \mu \lambda_{j}\right) \lambda_{j}}{\left(1+\tau \lambda_{j}\right)\left(1+(\tau+\mu) \lambda_{j}\right)} t} W_{j}(0),
$$

which is the desired estimate.

As a consequence we obtain, after the usual summation over $j$ (Fourier series), the following energy estimate for the energy term

$$
E^{\mu, \tau}(t):=\left\|\left(u_{t}, \mu \nabla u_{t}, \Delta u, \theta, \tau q\right)(t, \cdot)\right\|_{L^{2}(\Omega)}^{2} \cdot
$$

Theorem 5.2. There is a constant $C>0$ (in particular not depending on the parameters $\mu, \tau$ nor on the data), and a constant $k(\mu, \tau) \geq 0$, at most depending on $\mu, \tau$, such that for all $t \geq 0$ the estimate

$$
E^{\mu, \tau}(t) \leq C \mathrm{e}^{-c_{1} k(\mu, \tau) t} E^{\mu, \tau}(0)
$$

holds, where

$$
k(\mu, \tau):=\inf \left\{\varrho_{j}(\mu, \tau) \mid j \in \mathbb{N}\right\}
$$

and

$$
c_{1}:=\frac{1}{2370} .
$$

The value of $c_{1} k(\mu, \tau)$ is the estimate for the rate of exponential decay (if not zero) that we aimed at. We already mention the interesting fact that this analytical estimate is rather sharp, as a comparison with the numerical results from Section 7 will show.

Before we start determining $k(\mu, \tau)$ in more detail for $\mu, \tau>0$, we recall some known results for $\varrho_{j}$ for the limiting cases, see [19], then implying immediately the estimate for $k(\mu, \tau)$ in Table 5.1 . 


\begin{tabular}{|c|c|c|c|c|}
\hline case & $\mu, \tau$ & $\varrho_{j}$ & $k(\mu, \tau)$ & exp. stability $\mathrm{y} / \mathrm{n}$ \\
\hline \hline \hline $1:$ & $\mu=\tau=0$ & $\lambda_{j}$ & $\lambda_{1}$ & yes \\
\hline $2:$ & $\mu=0, \tau>0$ & $\frac{\lambda_{j}}{\left(1+\tau \lambda_{j}\right)^{2}}$ & 0 & no \\
\hline $3:$ & $\mu>0, \tau=0$ & $\frac{\lambda_{j}}{1+\mu \lambda_{j}}$ & $\frac{\lambda_{1}}{1+\mu \lambda_{1}}$ & yes \\
\hline
\end{tabular}

Table 5.1

The last column both describes the known facts on exponential stability of the associated semigroups, and the conclusion we draw from computing $k(\mu, \tau)$. In all cases $1-3$ these two things completely fit together.

We also observe the following facts on two singular limits and the ratio of exponential decay rates for the system with resp. without inertia term.

Remark 5.3. 1. The values $k(0, \tau)=0$ do not converge to $k(0,0)=\lambda_{1}>0$, as $\tau \rightarrow 0$.

2. The values $k(\mu, 0)=\frac{\lambda_{1}}{1+\mu \lambda_{1}}$ converge to $k(0,0)=\lambda_{1}$, as $\mu \rightarrow 0$.

3. If $\mu>0$, then

$$
k(\mu, 0)=\frac{\lambda_{1}}{1+\mu \lambda_{1}}<\lambda_{1}=k(0,0),
$$

i.e., the rate of decay is smaller for the system (with Fourier's law) with inertial term compared to that of the system without inertia term. The inertia term causes a slight deceleration (though both systems are exponentially stable).

Now we determine the value of $k(\mu, \tau)$ for $\mu, \tau>0$. Let

$$
g(x):=\frac{x(1+\tau \mu x)}{(1+\tau x)(1+(\tau+\mu) x)}, \quad x \geq \lambda_{1} .
$$

Remark 5.4. Depending on $\Omega, \lambda_{1}=\lambda_{1}(\Omega)$ can be close to zero or very large, cp. $\Omega=$ $(0, L) \subset \mathbb{R}^{1}: \lambda_{1}=\frac{\pi^{2}}{L^{2}} \rightarrow\left\{\begin{array}{c}0 \\ \infty\end{array}\right\}$ as $L \rightarrow\left\{\begin{array}{c}\infty \\ 0\end{array}\right\}$ 
The positive function $g$ satisfies

$$
\lim _{x \rightarrow \infty} g(x)=\frac{\mu}{(\tau+\mu)} \in(0,1) .
$$

To determine the infimum/minimum of $g$, we compute

$$
\begin{aligned}
g^{\prime}(x) & =\left\{\frac{1}{((1+\tau x)(1+(\tau+\mu) x))^{2}}\right\}\left\{\left[\tau\left(2 \tau \mu+\mu^{2}-\tau-\mu\right] x^{2}+2 \tau \mu x+1\right\}\right. \\
& \equiv\left\{g_{1}(x)\right\}\left\{g_{2}(x)\right\} .
\end{aligned}
$$

The coefficient [...] in front of $x^{2}$ in $g_{2}$ can be zero (e.g. $\tau=\frac{1}{4}, \mu=\frac{1+\sqrt{5}}{4} \approx 0.81$ ), positive (e.g. $\tau=\frac{1}{4}, \mu=0.9$ ), or negative (e.g. $\tau=\frac{1}{4}, \mu=0.8$ ), depending on $\tau, \mu$.

Case I: $\quad 2 \tau \mu+\mu^{2} \geq \tau+\mu$.

Then $g_{2}$ and thus $g^{\prime}$ are strictly positive, hence $g$ attains its minimum in $x=\lambda_{1}$, and we have

$$
k(\mu, \tau)=g\left(\lambda_{1}\right)=\frac{\lambda_{1}\left(1+\tau \mu \lambda_{1}\right)}{\left(1+\tau \lambda_{1}\right)\left(1+(\tau+\mu) \lambda_{1}\right)} .
$$

We remark already here, that for the limit $\mu, \tau \rightarrow 0$ this will not be the relevant case because of the quadratic nonlinearities becoming smaller than the linear ones.

Case II: $\quad 2 \tau \mu+\mu^{2}<\tau+\mu$.

In this case we have a zero $x_{0}=x_{0}^{\mu, \tau}$ of $g_{2}$ resp. $g^{\prime}$ at

$$
x_{0}^{\mu, \tau}=\frac{\tau \mu+\sqrt{\tau^{2} \mu^{2}+\tau\left(\tau+\mu-2 \tau \mu-\mu^{2}\right)}}{\tau\left(\tau+\mu-2 \tau \mu-\mu^{2}\right)} .
$$

Since, regarding $g$ on $(0, \infty)$ for a moment, $g^{\prime}(x)>0$ if $x<x_{0}^{\mu, \tau}$, and $g^{\prime}(x)<0$ if $x>x_{0}^{\mu, \tau}$, we have a local maximum of $g$ in $x_{0}^{\mu, \tau}$. So we have to distinguish the cases: $x_{0}^{\mu, \tau}<\lambda_{1}$ or $x_{0}^{\mu, \tau} \geq \lambda_{1}$. Both cases can happen since $x_{0}^{\mu, \tau}$ only depends on $\mu, \tau$, while $\lambda_{1}$ may take any value in $(0, \infty)$, depending on the domain $\Omega$, cp. Remark 5.4.

Case II.1: $\quad x_{0}^{\mu, \tau}<\lambda_{1}$.

In this case $g$ is strictly monotone decreasing on $\left[\lambda_{1}, \infty\right)$, thus the infimum of $g$ is attained at infinity, therefore

$$
k(\mu, \tau)=\lim _{x \rightarrow \infty} g(x)=\frac{\mu}{\tau+\mu} .
$$

Case II.2: $\quad x_{0}^{\mu, \tau} \geq \lambda_{1}$.

This case splits up into two final possible cases:

Case II.2.a: $\quad g\left(\lambda_{1}\right)<\lim _{x \rightarrow \infty} g(x)$.

Then

$$
k(\mu, \tau)=g\left(\lambda_{1}\right)=\frac{\lambda_{1}\left(1+\tau \mu \lambda_{1}\right)}{\left(1+\tau \lambda_{1}\right)\left(1+(\tau+\mu) \lambda_{1}\right)} .
$$


Case II.2.b: $\quad g\left(\lambda_{1}\right) \geq \lim _{x \rightarrow \infty} g(x)$.

Then

$$
k(\mu, \tau)=\lim _{x \rightarrow \infty} g(x)=\frac{\mu}{\tau+\mu} .
$$

Since we have the equivalencies

$$
\begin{aligned}
g\left(\lambda_{1}\right) & \leq \lim _{x \rightarrow \infty} g(x) \\
& \Longleftrightarrow \frac{\mu}{\tau+\mu} \\
\frac{\lambda_{1}\left(1+\tau \mu \lambda_{1}\right)}{\left(1+\tau \lambda_{1}\right)\left(1+(\tau+\mu) \lambda_{1}\right)} & \leq \frac{\mu}{\tau+\mu-2 \tau \mu-\mu^{2}}=: x_{1}^{\mu, \tau}, \\
\lambda_{1} & \leq \frac{\mu}{\tau+\mu}
\end{aligned}
$$

we can summarize the characterization of the decay rates $k(\mu, \tau)$ given in $(5.22)-(5.26)$ in the following theorem.

Theorem 5.5. The estimates for $k(\mu, \tau)$, for $\mu, \tau>0$, are given in Table 5.2, where $x_{0}^{\mu, \tau}$ is given in (5.23), and $x_{1}^{\mu, \tau}$ is given in (5.27).

In the cases I and II.2.a, for fixed $\mu, \tau$, the estimate on the decay rate shows a dependence on the first, the smallest eigenvalues $\lambda_{1}$, an effect that is known for the classical heat equation, and also showed up in Table 5.1 in the cases 2 and 3 . This is an effect of the notion of exponential stability which tries to be uniform over all initial values. Of course, if one has initial data taking values in subspaces not being spanned by the first eigenfunctions $\varphi_{1}, \ldots, \varphi_{k}$ (in other words: the expansions in (5.2) start at $j=k$ ), then $\lambda_{1}$ can be exchanged by $\lambda_{k}$ in these estimates. 


\begin{tabular}{|l|c|}
\hline \multicolumn{1}{|c|}{ case } & $k(\mu, \tau)$ \\
\hline \hline I: $2 \tau \mu+\mu^{2} \geq \tau+\mu$ & $\frac{\lambda_{1}\left(1+\tau \mu \lambda_{1}\right)}{\left(1+\tau \lambda_{1}\right)\left(1+(\tau+\mu) \lambda_{1}\right)}$ \\
\hline II.1: $2 \tau \mu+\mu^{2}<\tau+\mu, \quad \lambda_{1}>x_{0}^{\mu, \tau}$ & $\frac{\mu}{\tau+\mu}$ \\
\hline II.2.a: $2 \tau \mu+\mu^{2}<\tau+\mu, \quad \lambda_{1} \leq \min \left\{x_{0}^{\mu, \tau}, x_{1}^{\mu, \tau}\right\}$ & $\frac{\lambda_{1}\left(1+\tau \mu \lambda_{1}\right)}{\left(1+\tau \lambda_{1}\right)\left(1+(\tau+\mu) \lambda_{1}\right)}$ \\
\hline II.2.b: $2 \tau \mu+\mu^{2}<\tau+\mu, \quad x_{1}^{\mu, \tau}<\lambda_{1} \leq x_{0}^{\mu, \tau}$ & $\frac{\mu}{\tau+\mu}$ \\
\hline
\end{tabular}

Table 5.2
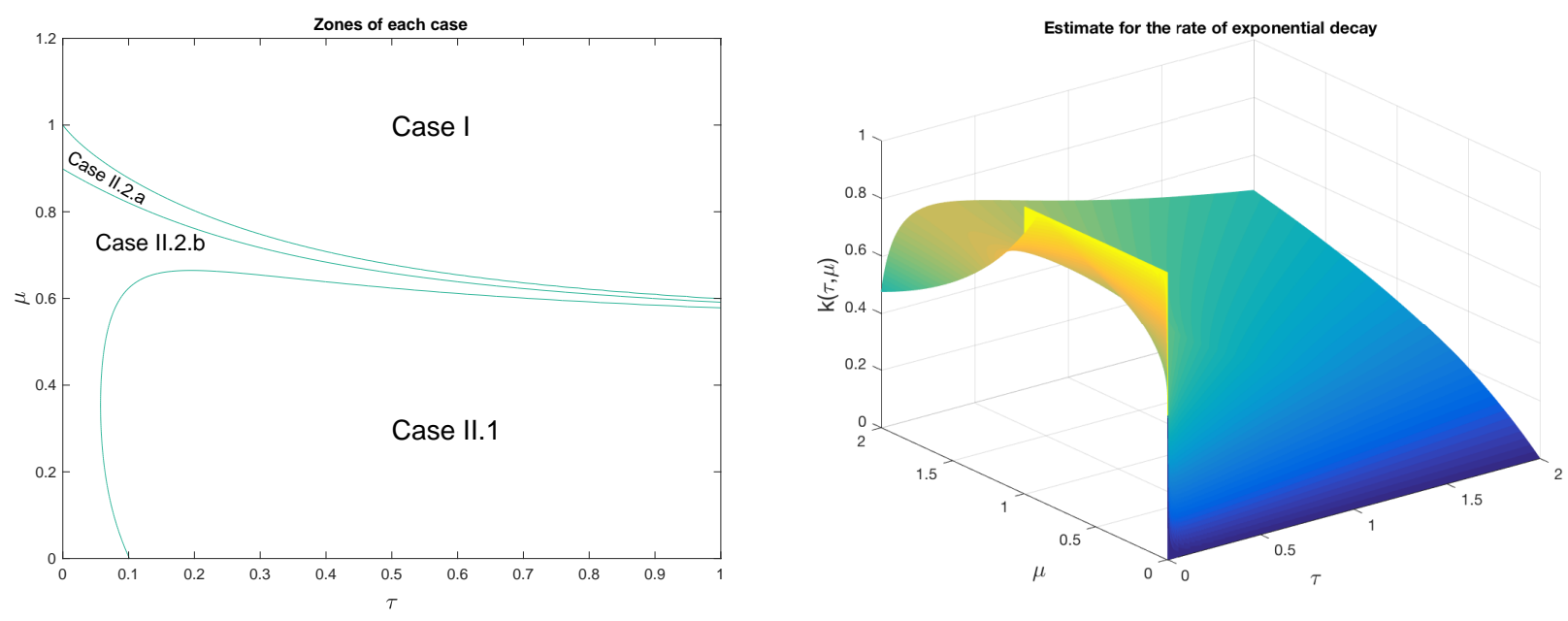

Figure 5.1: Estimate for the rate of exponential decay, and the 4 different cases zones of the Table $5.2(L=1)$.

\section{The limits $\mu \rightarrow 0$ and $\tau \rightarrow 0$ once more}

The characterizations of the decay rates $k(\mu, \tau)$ easily lead to a consideration of the singular limits $\mu, \tau \rightarrow 0$. Case I in Table 5.2 does now not play any role for the double 
limit since the quadratic terms in $\mu, \tau$ will finally be smaller than the linear ones. We have the following limit behavior.

Theorem 6.1. 1. If $\tau>0$, then only the cases II.1 and II.2.b are relevant. Here we obtain

$$
\lim _{\mu \rightarrow 0} k(\mu, \tau)=0
$$

2. If $\mu>0$, then case II.1 is not relevant. We obtain

$$
\lim _{\tau \rightarrow 0} k(\mu, \tau)=\left\{\begin{array}{cl}
1 & \text { in cases I and II.2.a }, \\
\frac{\lambda_{1}}{1+\mu \lambda_{1}}=k(\mu, 0) & \text { in case II.2.b. }
\end{array}\right.
$$

3.

$$
\lim _{\tau \rightarrow 0} \lim _{\mu \rightarrow 0} k(\mu, \tau)=0 \quad \text { in cases II.1 and II.2.b. }
$$

4. For domains $\Omega$ with $\lambda_{1} \leq 1$ we have

$$
\lim _{\mu \rightarrow 0} \lim _{\tau \rightarrow 0} k(\mu, \tau)=\lambda_{1}=k(0,0) \quad \text { in case II.2.a. }
$$

5. For domains $\Omega$ with $\lambda_{1} \geq 1$ we have

$$
\lim _{\mu \rightarrow 0} \lim _{\tau \rightarrow 0} k(\mu, \tau)=1 \quad \text { in case II.2.b. }
$$

Proof: The limits themselves can easily be obtained from Table 5.2. So we only comment on the restrictions made in points 1.-4.

1. Case I is not relevant because $2 \tau \mu+\mu^{2} \geq \tau+\mu$, but $\mu \rightarrow 0$. Case II.2.a is not relevant because $0<\lambda_{1} \leq x_{1}^{\mu, \tau}$, but $\lim _{\mu \rightarrow 0} x_{1}^{\mu, \tau}=0$.

2. Case II.1 is not relevant because $\infty>\lambda_{1}>x_{0}^{\mu, \tau}$, but $\lim _{\tau \rightarrow 0} x_{0}^{\mu, \tau}=\infty$.

4. resp. 5. The restriction $\lambda_{1} \leq 1$ resp. $\lambda_{1} \geq 1$ arises from $\lim _{\tau \rightarrow 0} x_{1}^{\mu, \tau}=\frac{1}{1-\mu} \rightarrow 1$, as $\mu \rightarrow 0$.

Theorem 6.1 shows once more that the function $k=k(\mu, \tau)$ is not continuous on $[0,1]^{2}$ (as well as $\left.x_{1}^{\mu, \tau}\right)$, reflecting the singular behavior, as $\mu, \tau \rightarrow 0$, of the property of exponential stability.

\section{Numerical analysis}

In this section, we present numerical results illustrating the asymptotic behavior of the energy and as well as the relevance of the condition of the Table 5.2 for the exponential decay. It yields an astonishing coincidence with the analytic estimates obtained in Theorem 5.1 above and in [19, Theorem 4.1], respectively. 
The applied method of $\beta$-Newmark is a second-order method preserving the discrete energy always when the discrete system of equations of motion is symmetric (i.e. matrices associated to the system should be symmetric).

\subsection{Shape and behavior of the spectrum}

In this section, we present some numerical results illustrating the asymptotic behavior of the energy and as well as the relevance of the condition of the Table 5.2 for the exponential decay. Here, we study the decay of the energy numerically. For this, we consider the approximation (5.2) and the systems of ODEs (5.3)-(5.5). This system can be rewritten as $\mathbf{X}_{j}^{\prime}=\mathbf{A}_{j} \mathbf{X}_{j}$, with $\mathbf{X}_{j}=\left(a_{j}, \dot{a}_{j}, b_{j}, f_{j}\right)^{T}$, and

$$
\mathbf{A}_{j}=\left(\begin{array}{cccc}
0 & 1 & 0 & 0 \\
-\frac{\lambda_{j}^{2}}{1+\mu \lambda_{j}} & 0 & \frac{\lambda_{j}}{1+\mu \lambda_{j}} & 0 \\
0 & -\lambda_{j} & 0 & \lambda_{j} \\
0 & 0 & \frac{-1}{\tau} & \frac{-1}{\tau}
\end{array}\right)
$$

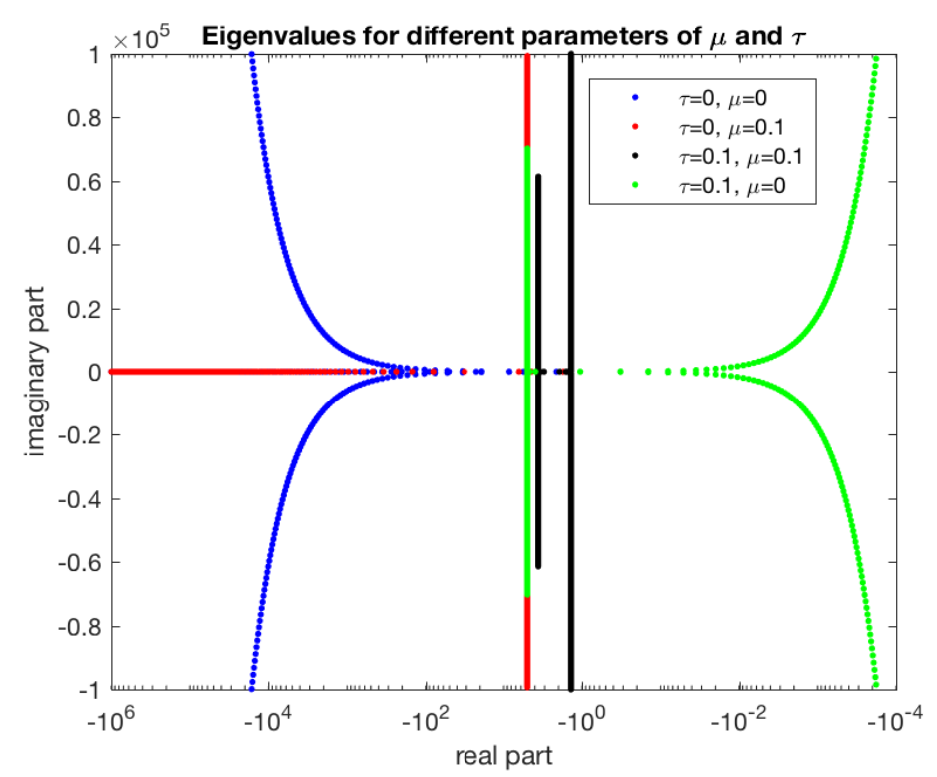

Figure 7.2: Eigenvalues of the system (1.1)-(1.3), with the ansatz (5.2), for different values of the parameters $\mu$ and $\tau$.

In the case of $\tau=0$, the Cattaneo system becomes the heat equation with the Fourier law (2.2), and therefore the $4 \times 4$ matrix of the system (7.1) (with 2 pairs of conjugate complex eigenvalues), is replaced by a $3 \times 3$ matrix with a pair of conjugate eigenvalues 
and a negative real value given by

$$
\mathbf{A}_{j}^{0}=\left(\begin{array}{ccc}
0 & 1 & 0 \\
-\frac{\lambda_{j}^{2}}{1+\mu \lambda_{j}} & 0 & \frac{\lambda_{j}}{1+\mu \lambda_{j}} \\
0 & -\lambda_{j} & -\lambda_{j}
\end{array}\right)
$$

The eigenvalues $\sigma_{j}^{\ell}$, of the matrix $\mathbf{A}_{j}$ defined in (7.1), with $\ell=1, \ldots, 4$, when $\tau>0$ (respectively $\mathbf{A}_{j}^{0}$ defined in (7.2), with $\ell=1,2,3$, when $\tau=0$ ), and $j=1, \ldots, \infty$, characterize all the eigenvalues of the system. In Figure 7.2, the eigenvalues (point spectrum) is plotted for different values of $\mu$ and $\tau$.

We consider the one-dimensional case $\Omega=(0, L)$ with $L=1$. Then $\lambda_{j}=j^{2} \pi^{2}$, and $\sigma_{j}^{\ell}$ are the roots of

$$
\tau \sigma^{4}+\sigma^{3}+\left(j^{2} \pi^{2}+2 \tau \frac{j^{4} \pi^{4}}{1+\mu j^{2} \pi^{2}}\right) \sigma^{2}+2 \frac{j^{4} \pi^{4}}{1+\mu j^{2} \pi^{2}} \sigma+\frac{j^{6} \pi^{6}}{1+\mu j^{2} \pi^{2}}=0 .
$$

An approximation of the rate of the exponential decay could be computed by

$$
\widetilde{k}(\mu, \tau)=\min _{j=1, \ldots, \infty}\left\{\left|\operatorname{Re}\left(\sigma_{j}^{\ell}\right)\right| / \ell=1, \ldots, 4(\text { if } \tau>0) ; \ell=1, \ldots, 3(\text { if } \tau=0)\right\} .
$$

$\widetilde{k}$ qualitatively should have the same behavior as the decay rate $k$ defined in (5.20), which is justified by Figure 7.2. Thus, we truncate $j=1, \ldots, N$, with $N=10.000$, in order to simulate the spectrum for different values of $\tau$ and $\mu$, and we show the graphs for $-10^{6}<\operatorname{Re}\left(\sigma_{j}^{\ell}\right)<0$ and $-10^{5}<\operatorname{Im}\left(\sigma_{j}^{\ell}\right)<10^{5}$. The real part of the eigenvalues in Figure 7.2 are plotted on a logarithmic scale so that all eigenvalues for different values of $\mu$ and $\tau$ can be drawn in the same graph ranging from $-10^{6}$ to $-10^{-4}$. In this sense, we observe that $(\mu=0$ and $\tau>0$ ) is the case where the eigenvalues are closer to the imaginary axis (taking into account the logarithmic scale), and these values approach the axis as $\lambda_{j} \rightarrow \infty$ (green eigenvalues in Figure 7.2). In this last case, the rate of the exponential decay is zero. In Table 7.1 we compare the values of $k(\mu, \tau)$ with $\widetilde{k}(\mu, \tau)$ for the different plotted cases in Figure 7.2.

\begin{tabular}{l||c|c|c|c} 
Case & $\tau=0 / \mu=0$ & $\tau=0 / \mu=0.1$ & $\tau=0.1 / \mu=0.1$ & $\tau=0.1 / \mu=0$ \\
\hline$k(\mu, \tau)$ & 1.000 & 1.000 & 0.500 & 0.000 \\
\hline$\widetilde{k}(\mu, \tau)$ & 2.1228 & 1.7430 & 1.3820 & $1.2665 \cdot 10^{-8}$
\end{tabular}

Table 7.1: Values of $k(\mu, \tau)$ and $\widetilde{k}(\mu, \tau)$ for the different plotted cases.

The approximation (5.2), suggests us to consider now also numerically Fourier series with respect to the spatial variable in dimension 1 , for an interval $\Omega=(0, L)$. On the 
other hand, regarding the approximation in time, we considers the $\beta$-Newmark scheme, which is a second order method preserving the discrete energy always when the discrete system of equations of motion is symmetric (i.e. matrices associated to the system should be symmetric).

Remark 7.1 (Finite Difference Approximation). With respect to an approximation using Finite Differences in the space variable, or another equivalent scheme (such as Finite Volumes or the Finite Element method), it is noticed that the eigenvalues undergo for very high frequencies. For example, if we consider $h=L /(J+1)$ a spatial subdivision of the interval $(0, L)$, with $x_{j}=j h$, and $u_{j}(t), \theta_{j}(t)$ and $q_{j}(t)$, for all $j=1,2, \ldots, J$, the approximate values of $u(j h, t), \theta(j h, t)$ and $q(j h, t)$, respectively for all $t>0$. Taking into account, centered finite differences of second order:

$$
\begin{gathered}
\Delta_{h} \vartheta_{j}:=\frac{\vartheta_{j+1}-2 \vartheta_{j}+\vartheta_{j-1}}{h^{2}}, \quad \Delta_{h}^{2} \vartheta_{j}:=\frac{\vartheta_{j+2}-4 \vartheta_{j+1}+6 \vartheta_{j}-4 \vartheta_{j-1}+\vartheta_{j-2}}{h^{4}}, \\
\delta_{h} \vartheta_{j}:=\frac{\vartheta_{j+1}-\vartheta_{j-1}}{2 h} .
\end{gathered}
$$

then the system (1.1)-(1.3) becomes an approximate system of $4 J \times 4 J$ ODEs when

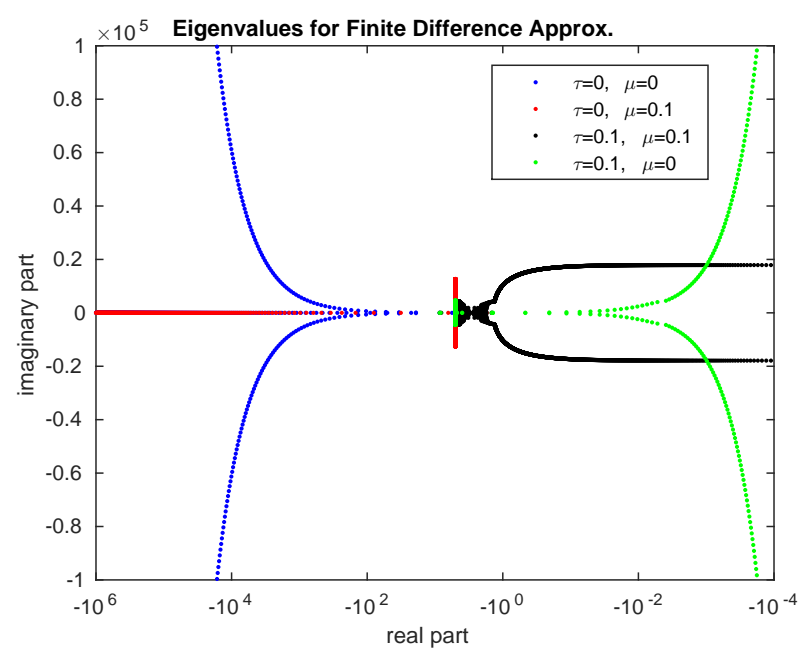

Figure 7.3: Eigenvalues of the Finite Difference system (7.3), for different values of the parameters $\mu$ and $\tau(J=2000)$.

$\tau>0$ (and a suitable system of $3 J \times 3 J$ ODEs when $\tau=0$ ) $\mathbf{Y}^{\prime}=\mathbf{B Y}$, with $\mathbf{Y}=$ $\left(u_{1}, \ldots, u_{J}, v_{1}, \ldots, v_{J}, \theta_{1}, \ldots, \theta_{J}, q_{1}, \ldots, q_{j}\right)^{T}$ when $\tau>0$ (and a system of $2 J \times 2 J$ ODEs 
when $\tau=0$ ), where

$$
\mathbf{B}=\left(\begin{array}{cccc}
\mathbf{O} & \mathbf{I} & \mathbf{O} & \mathbf{O} \\
-\left(\mathbf{I}-\mu \Delta_{h}\right)^{-1} \Delta_{h}^{2} & \mathbf{O} & -\left(\mathbf{I}-\mu \Delta_{h}\right)^{-1} \Delta_{h} & \mathbf{O} \\
\mathbf{O} & \Delta_{h} & \mathbf{O} & -\delta_{h} \\
\mathbf{O} & \mathbf{O} & \frac{-1}{\tau} \delta_{h} & \frac{-1}{\tau} \mathbf{I}
\end{array}\right)
$$

where $\mathbf{O}$ is the null matrix, and $\mathbf{I}$ is the identity.

We observe in Figure 7.3 that the eigenvalues of the approximate $\mathbf{B}$ matrix do not have precisely the same behavior as the eigenvalues of Figure 7.3. In particular, when $\tau=0.1$ and $\mu=0.1$, we observe that the approximate eigenvalues for finite differences adhere to the imaginary axis from sufficiently high frequencies (black graph in Figure 7.3), despite the fact that we know theoretically that it is one of the cases of exponential decay (compare with the equivalent graph in figure 7.2). Obviously, the approach method can be improved or equivalent formulations can be used for system (1.1)-(1.3), which under approximation, the eigenvalues are obtained more precisely and do not adhere to the axis $\operatorname{Re}(\lambda)=0$. However, for our numerical examples in dimension 1, the Fourier Series approximation is sufficiently efficient and accurate, and that is what we will use here.

\subsection{Time discretization for the equations of motion}

As the time discretization is concerned, it is desirable that the algorithm also has at least a second-order consistency, and because the spatial discretization used in structural dynamics often leads to inclusion of high-frequency modes in the model, it is also desirable to have unconditional stability. The method consists in updating the displacement, velocity and acceleration vectors from current time $t^{n}=n \delta t$ to the time $t^{n+1}=(n+1) \delta t$. The Newmark algorithm [15] is based on a set of two relations expressing the forward displacement $u_{\delta}^{n+1}$ and velocity $\dot{u}_{\delta}^{n+1}$ in terms of their current values and the forward and current values of the acceleration. In the case of the variable $u(t, x)=\sum_{j=1}^{\infty} a_{j}(t) \sin \frac{j \pi x}{L}$, we have

$$
\begin{aligned}
& \dot{a}_{j}^{n+1}=\dot{a}_{j}^{n}+(1-\gamma) \delta t \ddot{a}_{j}^{n}+\gamma \delta t \ddot{a}_{j}^{n+1} \\
& a_{j}^{n+1}=a_{j}^{n}+\delta t \dot{a}_{j}^{n}+\left(\frac{1}{2}-\beta\right) \delta t^{2} \ddot{a}_{j}^{n}+\beta \delta t^{2} \ddot{a}_{j}^{n+1}
\end{aligned}
$$

where $\beta$ and $\gamma$ are parameters of the methods that will be fixed later. Replacing (7.4), (7.5) in the equation of motion (5.3), we obtain

$$
\begin{aligned}
\left(1+\mu \lambda_{j}+\beta \delta t^{2} \lambda_{j}^{2}\right) & \ddot{a}_{j}^{n+1}-\lambda_{j} b_{j}^{n+1} \\
& =-\lambda_{j}^{2}\left(a_{j}^{n}+\delta t \dot{a}_{j}^{n}+\left(\frac{1}{2}-\beta\right) \delta t^{2} \ddot{a}_{j}^{n}\right) .
\end{aligned}
$$




\subsection{Discretization of the heat equation}

The heat equation system (5.2), (5.3) can be approximated by trapezoidal rule

$$
\begin{aligned}
& b_{j}^{n+1}-b_{j}^{n}-\lambda_{j} \delta t f_{j}^{n+\frac{1}{2}}+\lambda_{j} \delta t \dot{a}_{j}^{n+\frac{1}{2}}=0, \\
& \tau\left(f_{j}^{n+1}-f_{j}^{n}\right)+\delta t f_{j}^{n+\frac{1}{2}}+\delta t b_{j}^{n+\frac{1}{2}}=0,
\end{aligned}
$$

where $b_{j}^{n+\frac{1}{2}}=\frac{b_{j}^{n}+b_{j}^{n+1}}{2}$ and $\dot{a}_{j}^{n+\frac{1}{2}}=\frac{\dot{a}_{j}^{n}+\dot{a}_{j}^{n+1}}{2}$. Then, replacing (7.4) in (7.7), we obtain

$$
\begin{aligned}
\gamma \delta t^{2} \frac{\lambda_{j}}{2} \ddot{a}_{j}^{n+1}+b_{j}^{n+1} & -\lambda_{j} \frac{\delta t}{2} f_{j}^{n+1} \\
& =b_{j}^{n}+\lambda_{j} \frac{\delta t}{2} f_{j}^{n}-\lambda_{j}\left(\delta t \dot{a}_{j}^{n}+(1-\gamma) \frac{\delta t^{2}}{2} \ddot{a}_{j}^{n}\right)
\end{aligned}
$$

The system of equation $(7.6),(7.8),(7.9)$, can be written in matrix form as

$$
\left(\begin{array}{ccc}
1+\mu \lambda_{j}+\beta \delta t^{2} \lambda_{j}^{2} & -\lambda_{j} & 0 \\
\gamma \delta t^{2} \frac{\lambda_{j}}{2} & 1 & -\lambda_{j} \frac{\delta t}{2} \\
0 & \lambda_{j} \frac{\delta t}{2} & \lambda_{j}\left(\tau+\frac{\delta t}{2}\right)
\end{array}\right)\left(\begin{array}{c}
\ddot{a}_{j}^{n+1} \\
b_{j}^{n+1} \\
f_{j}^{n+1}
\end{array}\right)=\left(\begin{array}{c}
\mathcal{A}_{j}^{n} \\
\mathcal{B}_{j}^{n} \\
\mathcal{F}_{j}^{n}
\end{array}\right)
$$

where $\mathcal{A}_{j}^{n}=-\lambda_{j}^{2}\left(a_{j}^{n}+\delta t \dot{a}_{j}^{n}+\left(\frac{1}{2}-\beta\right) \delta t^{2} \ddot{a}_{j}^{n}\right), \mathcal{B}_{j}^{n}=b_{j}^{n}+\lambda_{j} \frac{\delta t}{2} f_{j}^{n}-\lambda_{j}\left(\delta t \dot{a}_{j}^{n}+(1-\gamma) \frac{\delta t^{2}}{2} \ddot{a}_{j}^{n}\right)$, and $\mathcal{F}_{j}^{n}=\left(\tau-\frac{\delta t}{2}\right) f_{j}^{n}-\lambda_{j} \frac{\delta t}{2} b_{j}^{n}$.

Remark 7.2 (Case $\tau=0$.). The system of equation (7.6),(7.8), (7.9), is also valid when $\tau=0$. However, (7.8) is reduced to $f_{j}^{n+\frac{1}{2}}=-b_{j}^{n+\frac{1}{2}}$ and the system (7.10) is simplified to the $2 \times 2$ system

$$
\left(\begin{array}{cc}
1+\mu \lambda_{j}+\beta \delta t^{2} \lambda_{j}^{2} & -\lambda_{j} \\
\gamma \delta t^{2} \frac{\lambda_{j}}{2} & 1+\lambda_{j} \frac{\delta t}{2}
\end{array}\right)\left(\begin{array}{c}
\ddot{a}_{j}^{n+1} \\
b_{j}^{n+1}
\end{array}\right)=\left(\begin{array}{c}
\mathcal{A}_{j}^{n} \\
\widehat{\mathcal{B}}_{j}^{n}
\end{array}\right)
$$

where $\widehat{\mathcal{B}}_{j}^{n}=\left(1-\lambda_{j} \frac{\delta t}{2}\right) b_{j}^{n}-\lambda_{j}\left(\delta t \dot{a}_{j}^{n}+(1-\gamma) \frac{\delta t^{2}}{2} \ddot{a}_{j}^{n}\right)$.

\subsection{Decay of the discrete energy}

The eigenfunctions of the Dirichlet Laplace operator $-\Delta$ in $\Omega=(0, L)$ are given by $\phi_{j}(x)=\sin \frac{j \pi x}{L}$. Therefore, the discrete energy can be written as

$$
E^{n}=\sum_{j=1}^{\infty}\left(1+\mu \lambda_{j}\right)\left|\dot{a}_{j}^{n}(t)\right|^{2}+\lambda_{j}^{2}\left|a_{j}^{n}\right|^{2}+\left|b_{j}^{n}\right|^{2}+\tau \lambda_{j}\left|f_{j}^{n}\right|^{2}
$$

The decrease of the energy of time $t_{n}$ at time $t_{n+1}$ can be expressed in terms of the mean value and the increments of the displacements and the velocity by the following identity

$$
E^{n+1}-E^{n}=\sum_{j=1}^{\infty}\left[\left(1+\mu \lambda_{j}\right)\left|\dot{a}_{j}(t)\right|^{2}+\lambda_{j}^{2}\left|a_{j}\right|^{2}+\left|b_{j}\right|^{2}+\tau \lambda_{j}\left|f_{j}\right|^{2}\right]_{n}^{n+1}
$$


where

$$
\begin{aligned}
\frac{1}{2}\left[\left(1+\mu \lambda_{j}\right)\left|\dot{a}_{j}(t)\right|^{2}\right. & \left.+\lambda_{j}^{2}\left|a_{j}\right|^{2}+\left|b_{j}\right|^{2}+\tau \lambda_{j}\left|f_{j}\right|^{2}\right]_{n}^{n+1} \\
= & \dot{a}_{j}^{n+\frac{1}{2}}\left(1+\mu \lambda_{j}\right) \Delta^{n} \dot{a}_{j}+a_{j}^{n+\frac{1}{2}} \lambda_{j}^{2} \Delta^{n} a_{j}+b_{j}^{n+\frac{1}{2}} \Delta^{n} b_{j} \\
& +f_{j}^{n+\frac{1}{2}} \tau \lambda_{j} \Delta^{n} f_{j}
\end{aligned}
$$

with the notation $\Delta^{n} u=u^{n+1}-u^{n}$. Then, expressing the Newmark representation formulae (7.4), (7.5) in term of increments and mean values

$$
\begin{aligned}
& \Delta^{n} \dot{a}_{j}=\delta t \ddot{a}_{j}^{n+\frac{1}{2}}+\left(\gamma-\frac{1}{2}\right) \delta t \Delta^{n} \ddot{a}_{j} \\
& \Delta^{n} a_{j}=\delta t \dot{a}_{j}^{n+\frac{1}{2}}+\left(\beta-\frac{1}{2} \gamma\right) \delta t^{2} \Delta^{n} \ddot{a}_{j}
\end{aligned}
$$

and replacing $(7.7),(7.8),(7.12)$ and (7.13), in (7.11), we obtain

$$
\begin{aligned}
\frac{1}{2}\left[\left(1+\mu \lambda_{j}\right)\left|\dot{a}_{j}(t)\right|^{2}\right. & \left.+\lambda_{j}^{2}\left|a_{j}\right|^{2}+\left|b_{j}\right|^{2}+\tau \lambda_{j}\left|f_{j}\right|^{2}\right]_{n}^{n+1} \\
& =\left(\left(1+\mu \lambda_{j}\right)\left(\gamma-\frac{1}{2}\right) \delta t \dot{a}_{j}^{n+\frac{1}{2}}+\lambda_{j}^{2}\left(\beta-\frac{1}{2} \gamma\right) \delta t^{2} a_{j}^{n+\frac{1}{2}}\right) \Delta^{n} \ddot{a}_{j} \\
& -\lambda_{j} \delta t\left|f_{j}^{n+\frac{1}{2}}\right|^{2}
\end{aligned}
$$

Then, by choosing $\gamma=\frac{1}{2}$ and $\beta=\frac{\gamma}{2}$, we deduce

$$
\frac{E^{n+1}-E^{n}}{\delta t}=-\sum_{j=1}^{\infty} \lambda_{j}\left|f_{j}^{n+\frac{1}{2}}\right|^{2} \leq 0
$$

That is, the energy is decreasing, in coherence with (3.4). We note that, when $\tau=0$, the right hand side term of $(7.15)$, must be replaced by $-\sum_{j=1}^{\infty} \lambda_{j}\left|b_{j}^{n+\frac{1}{2}}\right|^{2}$, which is consistent with (2.3).

\subsection{Numerical examples}

We consider the following initial condition for $u(x, t)$ :

$$
u(x, 0)=u_{0}(x)=1-2|x|, \quad \forall 0<x<L,
$$

$u_{t}(x, 0)=0, \theta(x, 0)=\sin \frac{\pi x}{L}$ and $q(x, 0)=\frac{\pi}{L} \cos \frac{\pi x}{L}$. Then, we make simulation for $L=1$, and we approximate (5.2) by

$$
u(t, x)=\sum_{j=1}^{N} a_{j}(t) \phi_{j}(x), \quad \theta(t, x)=\sum_{j=1}^{N} b_{j}(t) \phi_{j}(x), \quad q(t, x)=\sum_{j=1}^{N} f_{j}(t) \nabla \phi_{j}(x) .
$$




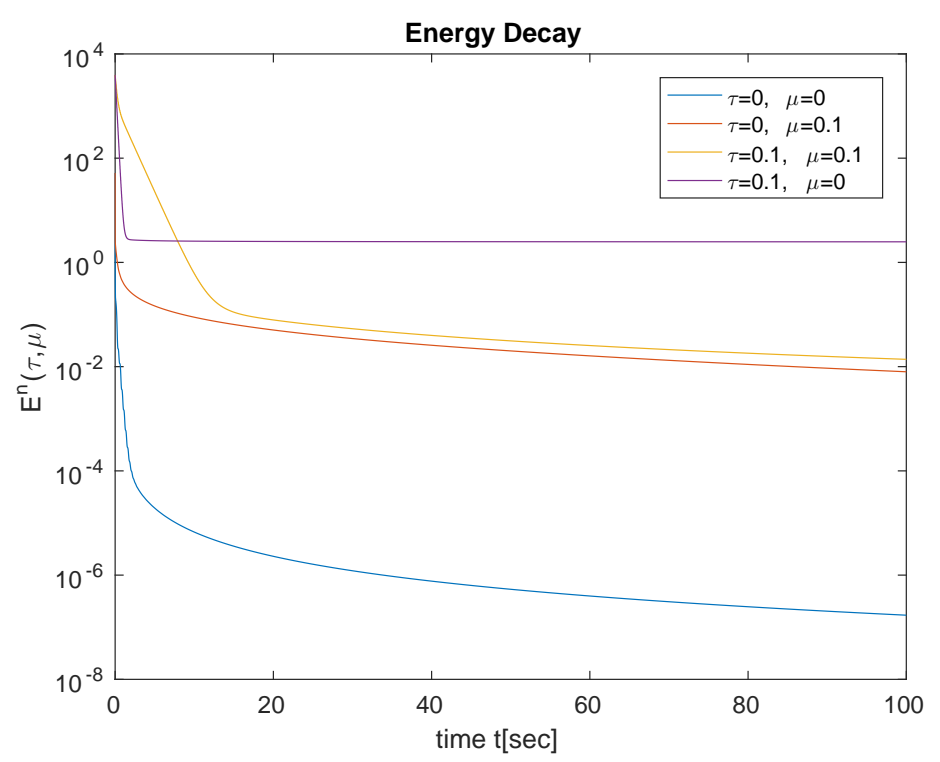

Figure 7.4: Energy decay for different values of $\mu$ and $\tau$,

with $N=1000$. We compare $(\mu=0, \tau=0),(\mu>0, \tau=0),(\mu=0, \tau>0)$ and $(\mu>0, \tau>0)$. For this, we make a simulation with a time discretization for $T=1000$, $N_{t}=100.000$ and $\delta t=T / N_{t}$. We observe in Figure 7.3 that the case $\mu=0, \tau=0$ is one that decays fastest. On the other hand, the case $\mu=0, \tau=1$ (the non-exponential decay case), decays so slowly with respect to the other three cases, at least in this graph in semilog scale.

Finally, in Figure 7.5 (case $\mu=0, \tau=0$ ) and in Figure 7.6 (case $\mu=0, \tau=1$ ), we observe the asymptotic behavior of the different variables $u(x, t), u_{t}(x, t), \theta(x, t)$ and $q(x, t)$ for differente values of $\mu$ and $\tau$. For the four cases considered in figure 7.3, we consider the most extreme cases, namely the one that decays the most rapidly (when $\mu=0$ and $\tau=0$ ), and the one that does not decay exponentially (when $\mu=0$ and $\tau=1$ ). In addition to the decay, we can observe that for the non-exponential case (Figure 7.6), the variables present greater oscillations in time, and in particular, the variable velocity $u_{t}(x, t)$ is that which decays more slowly.

\subsubsection{Comparison between theoretical and numerical rates}

Now we may compare some numerical results obtained with those given by the estimate in Table 5.2. It turns out that there are remarkable coincidences, also demonstrating that the analytic estimates which prove Theorem 5.1 - going back to the calculations in [19] for the Cauchy problem - are rather sharp.

We consider two examples: 

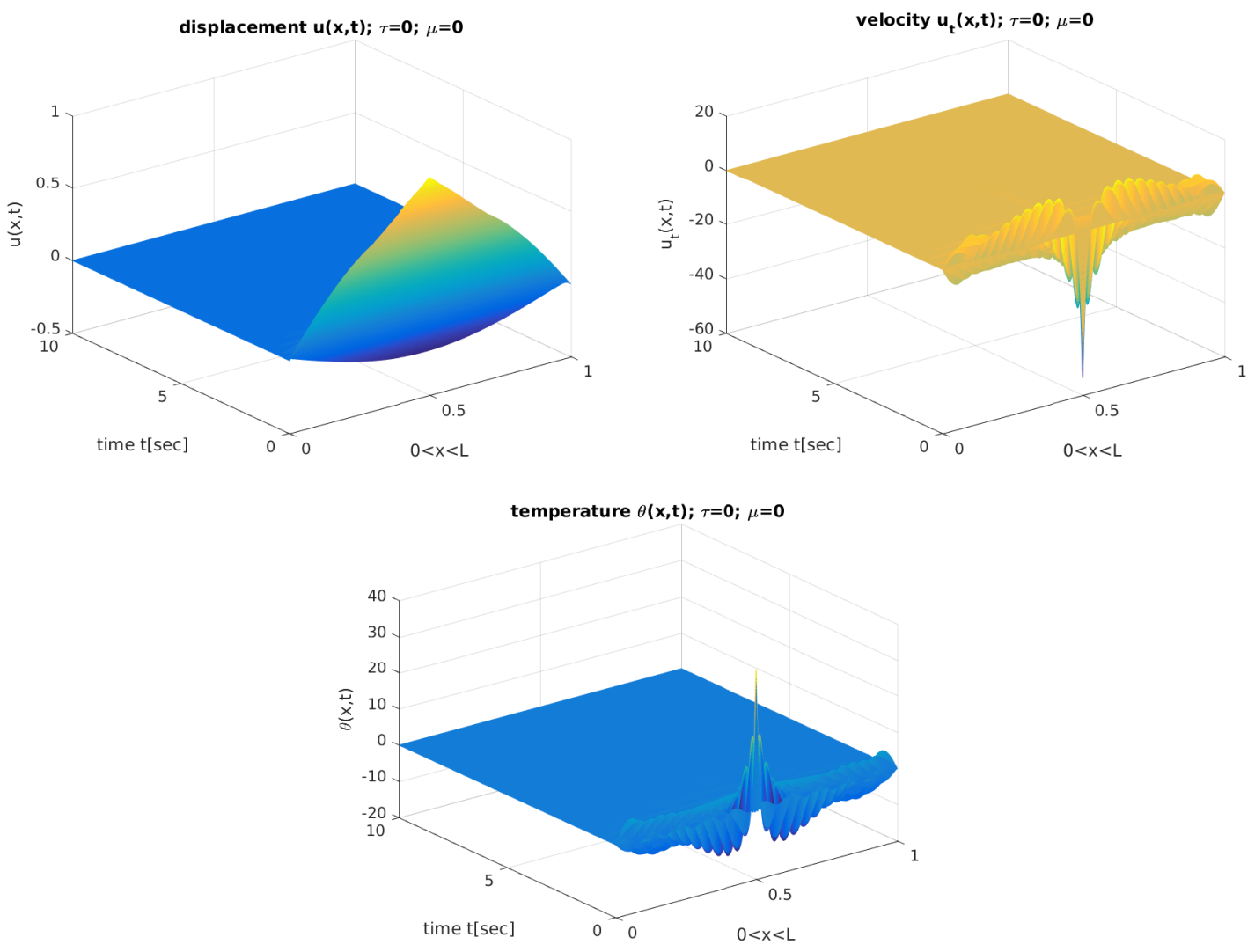

Figure 7.5: Asymptotic behavior of $u(x, t), u_{t}(x, t)$ and $\theta(x, t)$ for $\mu=0$ and $\tau=0$,

1. $\tau=0.1 ; \mu=0.5$ : Since $2 \tau \mu+\mu^{2}<\tau+\mu$, we are in Case II of Table 5.2. Computing

$$
x_{0}^{\mu, \tau}=\frac{\tau \mu+\sqrt{\tau^{2} \mu^{2}+\tau\left(\tau+\mu-2 \tau \mu-\mu^{2}\right)}}{\tau\left(\tau+\mu-2 \tau \mu-\mu^{2}\right)}=8.6333 \text {, }
$$

and

$$
x_{1}^{\mu, \tau}=\frac{\mu}{\tau+\mu-2 \tau \mu-\mu^{2}}=2.0
$$

we have $x_{1}^{\mu, \tau}<\pi^{2}=\lambda_{1}>x_{0}^{\mu, \tau}$. Hence, we are in Case II.1. Thus we get for the analytical estimate for the decay rate from Table 5.2

$$
r_{a}=c_{1} \frac{\mu}{\tau+\mu}=c_{1} \frac{10}{12}=3.0525 \cdot 10^{-4} .
$$

Using linear regression on the last 10 values of energy $E^{n}$ (for $\left.n=N-9, \ldots, N\right)$, we obtain that $E(t) \approx C_{n} e^{-r_{n} t}$, with

$$
r_{n}=4.861 \cdot 10^{-4}
$$

$C_{n}=0.3008$, and with the goodness of fit given by

$$
R^{2}=\frac{\sum_{n=1}^{N}\left(E^{n}-C_{n} e^{-r_{n} t_{n}}\right)}{\sum_{n=1}^{N}\left(E^{n}-\bar{E}^{n}\right)}=0.99998521 .
$$



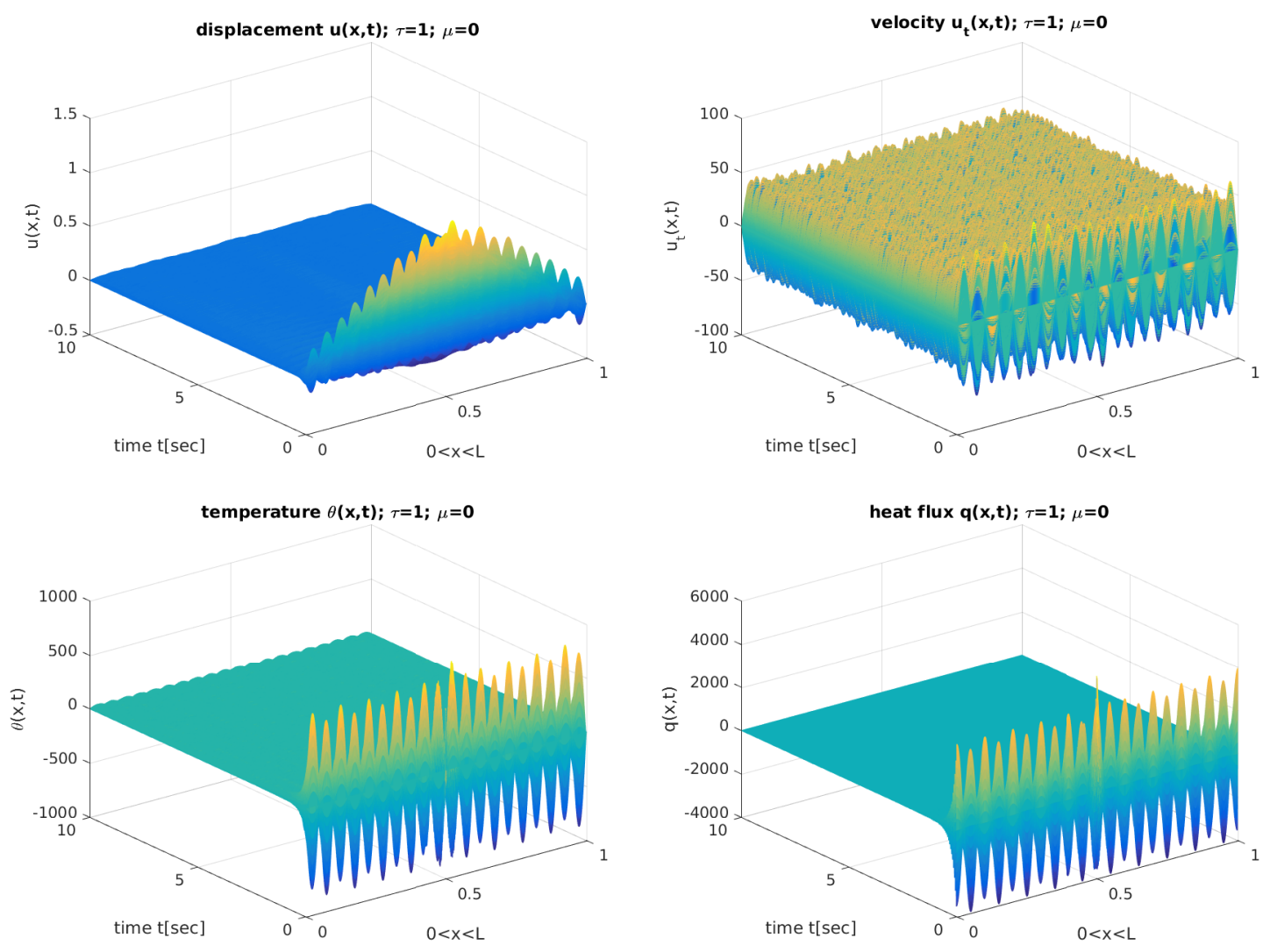

Figure 7.6: Asymptotic behavior of $u(x, t), u_{t}(x, t), \theta(x, t)$ and $q(x, t)$ for $\mu=0$ and $\tau=1$,

On the other hand, we have $C_{a}=\frac{E(t)}{e^{-r_{a} t}}=0.1218$, for $t=5000$. We compare both approximations (numerical and thoerical) in Figure 7.7. We have $r_{a} \leq r_{n}$ as expected, and in particular $r_{a} \approx r_{n}$ in an astonishingly (in view of the many seemingly "coarse" estimates in deriving Theorem 5.1) sharp sense.

2. $\tau=0.01, \mu=0.1$ : Since $2 \tau \mu+\mu^{2}<\tau+\mu$, we are in Case II of Table 5.2. Computing

$$
x_{0}^{\mu, \tau}=\frac{\tau \mu+\sqrt{\tau^{2} \mu^{2}+\tau\left(\tau+\mu-2 \tau \mu-\mu^{2}\right)}}{\tau\left(\tau+\mu-2 \tau \mu-\mu^{2}\right)}=\frac{1000}{9.8}=102.04,
$$

and

$$
x_{1}^{\mu, \tau}=\frac{\mu}{\tau+\mu-2 \tau \mu-\mu^{2}}=\frac{10}{9.8}=1.0204
$$

we have $x_{1}^{\mu, \tau}<\pi^{2}=\lambda_{1}<x_{0}^{\mu, \tau}$. Hence, we are in Case II.2.b. Thus we get for the analytical estimate for the decay rate from Table 5.2

$$
r_{a}=c_{1} \frac{\mu}{\tau+\mu}=c_{1} \frac{10}{11}=3.330 \cdot 10^{-4} .
$$




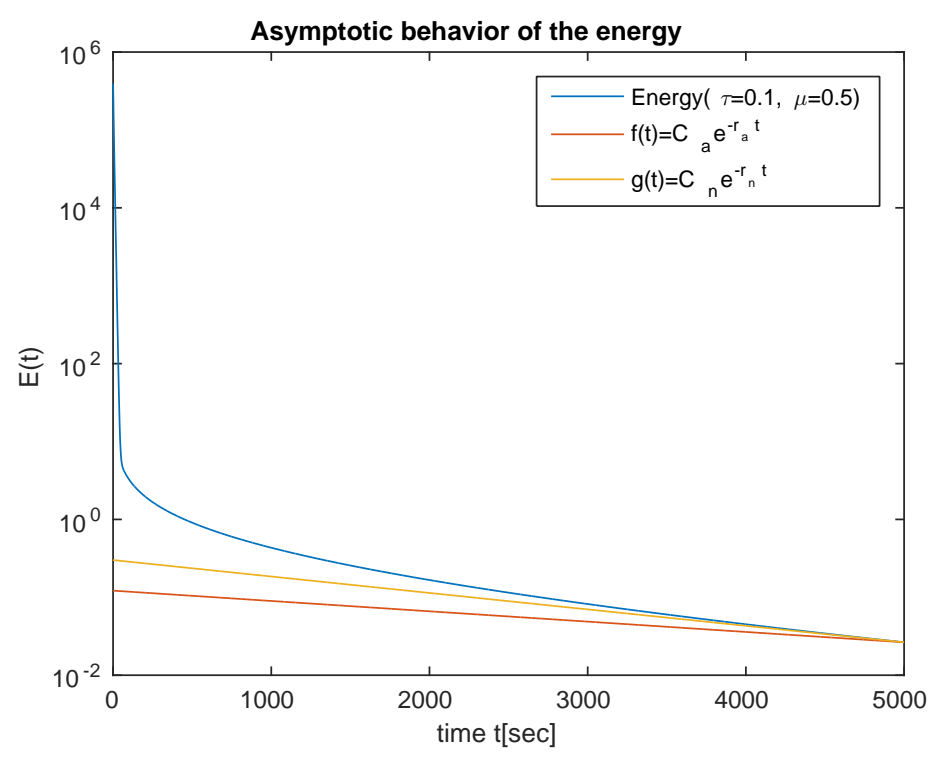

Figure 7.7: Example 1: asymptotic behavior of the energy for $\mu=0.5$ and $\tau=0.1$ compared with negative rate exponential functions $f(t)=C_{a} e^{-r_{a} t}$ (theoretical) and $g(t)=$ $C_{n} e^{-r_{n} t}$ (numerical).

The numerically obtained value for the decay rate $r_{n}$ using linear regression on the last 10 values of energy is

$$
r_{n}=5.1668 \cdot 10^{-4}
$$

$C_{n}=0.0883$, and with the goodness of fit given by $R^{2}=1.000$. Again we have $C_{a}=\frac{E(t)}{e^{-r_{a} t}}=0.0352$, for $t=5000$. We compare both approximations (numerical and thoerical) in Figure 7.8. We have that $r_{a} \leq r_{n}$ as expected, and $r_{a} \approx r_{n}$ underlining the analytical estimates.

\begin{tabular}{l||c|c|c|c|c} 
Rates & $k(\mu, \tau)$ & $r_{a}$ & $\widetilde{k}(\mu, \tau)$ & $\widetilde{r}_{a}$ & $r_{n}$ \\
\hline Example 1 & 0.83333 & $3.056 \cdot 10^{-4}$ & 0.9951 & $3.645 \cdot 10^{-4}$ & $4.861 \cdot 10^{-4}$ \\
\hline Example 2 & 0.90909 & $3.330 \cdot 10^{-4}$ & 1.949 & $7.139 \cdot 10^{-4}$ & $5.167 \cdot 10^{-4}$
\end{tabular}

Table 7.2: Different values of the rates for both examples: theoretical $r_{a}=c_{1} \cdot k(\mu, \tau)$; numerical by approximation of the eigenvalues $\widetilde{r}_{a}=c_{1} \cdot \widetilde{k}(\mu, \tau)$; and numerical by interpolation of the slope for the discrete energy $r_{n}$.

Finally, in Table 7.2, we summarize the different estimates of the rates, theoretical $\left(r_{a}\right)$, numerical $\left(r_{n}\right)$, and we also add a rate $\widetilde{r}_{a}=c_{1} \cdot \widetilde{k}(\mu, \tau)$, calculated using value of $\widetilde{k}$ instead of $k$. 


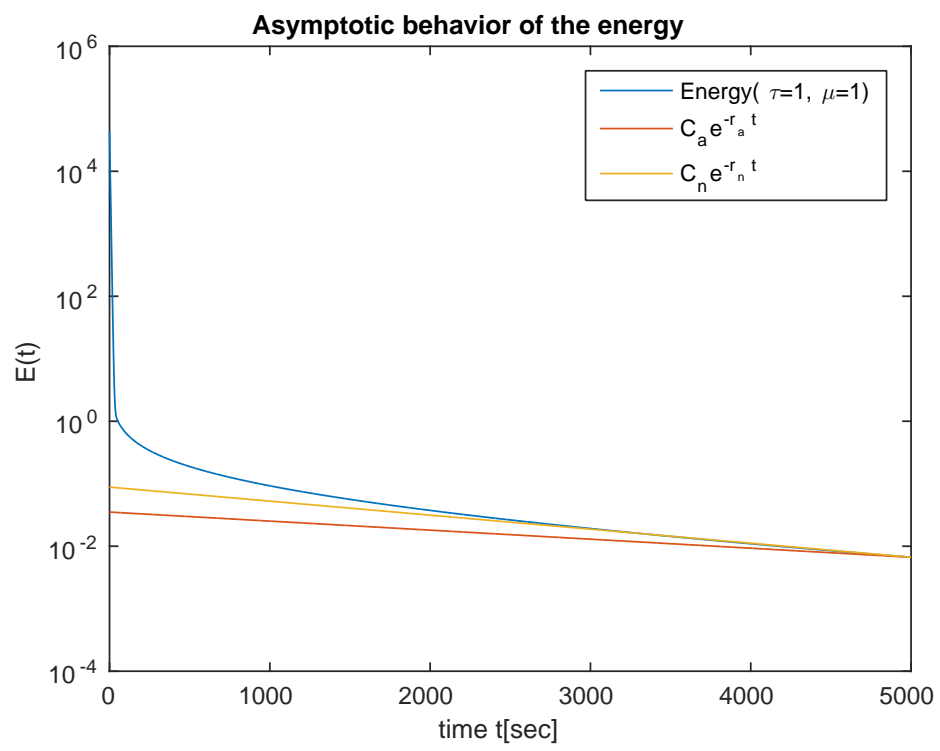

Figure 7.8: Example 2: asymptotic behavior of the energy for $\mu=0.1$ and $\tau=0.01$ compared with negative rate exponential functions $f(t)=C_{a} e^{-r_{a} t}$ (analytical) and $g(t)=$ $C_{n} e^{-r_{n} t}$ (numerical).

Acknowledgements: The authors have been supported by the Brazilian agency CNPq within the project "Ciências sem Fronteiras", grant \#402689/2012-7.

M. Sepúlveda thanks the support of Bolsa PCI-LNCC 300246/2015-3, FONDECYT grant no. 1180868, and Basal, CMM, Universidad de Chile and $\mathrm{CI}^{2} \mathrm{MA}$, Universidad de Concepción.

O.Vera thanks the support of Bolsa PCI-LNCC 2017.

\section{References}

[1] Avalos, G., Lasiecka, I.: Exponential stability of a thermoelastic system without mechanical dissipation. Rend. Instit. Mat. Univ. Trieste Suppl. 28 (1997), 1-28.

[2] Denk, R., Shibata, R.: Maximal regularity for the thermoelastic plate equations with free boundary conditions. J. Evolution Equations 17 (2017), 215-261.

[3] Fernández Sare, H.D., Muñoz Rivera, J.E.: Optimal rates of decay in 2-d thermoelasticity with second sound. J. Math. Phys. 53 (2012), 073509-1-13. 
[4] Fernández Sare, H.D., Racke, R.: On the stability of damped Timoshenko systems Cattaneo versus Fourier law. Arch. Rational Mech. Anal. 194 (2009), 221-251.

[5] Grasselli, M., Squassina, M.: Exponential stability and singular limit for a linear thermoelastic plate with memory effects. Adv. Math. Sci. Appl. 16 (2006), 15-31.

[6] Kim, J. U.: On the energy decay of a linear thermoelastic bar and plate. SIAM J. Math. Anal. 23 (1992), 889-899.

[7] Lasiecka, I., Triggiani, R.: Two direct proofs on the analyticity of the S.C. semigroup arising in abstract thermoelastic equations. Adv. Differential Equations 3 (1998), 387-416.

[8] Lasiecka, I., Triggiani, R.: Analyticity, and lack thereof, of thermo-elastic semigroups. ESAIM, Proc. 4 (1998), 199-222.

[9] Lasiecka, I., Triggiani, R.: Analyticity of thermo-elastic semigroups with coupled hinged/Neumann boundary conditions. Abstract Appl. Anal. 3 (1998), 153-169.

[10] Lasiecka, I., Triggiani, R.: Analyticity of thermo-elastic semigroups with free boundary conditions. Annali Scuola Norm. Sup. Pisa 27 (1998), 457-482.

[11] Lasiecka, I., Wilke, M.: Maximal regularity and global existence of solutions to a quasilinear thermoelastic plate system. Discrete Contin. Dyn. Syst. 33 (2013), 5189--5202.

[12] Liu, Z., Zheng, S.: Exponential stability of the Kirchhoff plate with thermal or viscoelastic damping. Quart. Appl. Math. 53 (1997), 551-564.

[13] Muñoz Rivera, J. E., Racke, R.: Smoothing properties, decay, and global existence of solutions to nonlinear coupled systems of thermoelastic type. SIAM J. Math. Anal. 26 (1995), 1547-1563.

[14] Muñoz Rivera, J.E., Racke, R.: Large solutions and smoothing properties for nonlinear thermoelastic systems. J. Differential Equations 127 (1996), 454-483.

[15] Newmark, N.M.: A method of computation for structural dynamics. J. Engrg. Mech. Div. $A S C E 85$ (1959), 67-94 .

[16] Naito, Y.: On the $L_{p}-L_{q}$ maximal regularity for the linear thermoelastic plate equation in a bounded domain. Math. Meth. Appl. Sci. 32 (2009), 1609--1637.

[17] Quintanilla, R., Racke, R.: Addendum to: Qualitative aspects of solutions in resonators. Arch. Mech. 63 (2011), 429-435.

[18] Racke, R.: Heat conduction in elastic systems: Fourier versus Cattaneo. Proc. International Conference on Heat Transfer, Fluid Mechanics and Thermodynamics, Skukuza, South Africa (2015), 356-360. 
[19] Racke, R., Ueda, Y.: Dissipative structures for thermoelastic plate equations in $\mathbb{R}^{n}$. Adv. Differential Equations 21 (2016), 601-630.

Jaime E. Muñoz Rivera, National Laboratory of Scientific Computation, Av. Getúlio Vargas 333, 25651-075 Petrópolis, Brazil

rivera@lncc.br

Reinhard Racke, Department of Mathematics and Statistics, University of Konstanz, 78457 Konstanz, Germany

reinhard.racke@uni-konstanz.de

Mauricio Sepúlveda, Department of Engineering, Faculty of Physics and Mathematics, University of Concepción, Casilla 160-C, Concepción, Chile

mauricio@ing-mat.udec.cl

Octavio Vera Villagrán, Department of Mathematics, University of Bío-Bío, Concepción, Chile overa@ubiobio.cl 